\title{
UPDATING THE FREIGHT TRUCK STOCK ADJUSTMENT MODEL: 1997 VEHICLE INVENTORY AND USE SURVEY DATA
}

\author{
Stacy C. Davis
}

November 2000

\author{
Prepared for the \\ Energy Information Administration \\ U.S. Department of Energy \\ Prepared by the \\ OAK RIDGE NATIONAL LABORATORY \\ Oak Ridge, Tennessee 37831-6073 \\ managed by \\ UT-BATTELLE, LLC \\ for the \\ U.S. DEPARTMENT OF ENERGY \\ under Contract No. DE-AC05-00OR22725
}





\section{TABLE OF CONTENTS}

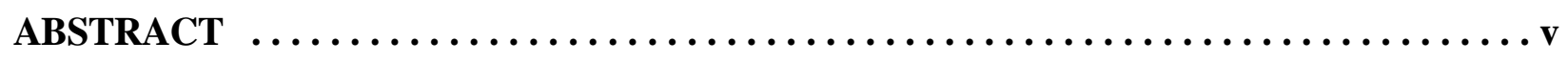

INTRODUCTION $\ldots \ldots \ldots \ldots \ldots \ldots \ldots \ldots \ldots \ldots \ldots \ldots \ldots \ldots \ldots \ldots \ldots \ldots \ldots$

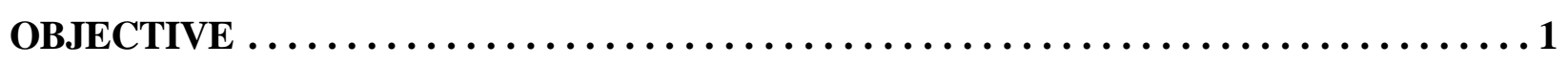

VIUS DATA PREPARATION $\ldots \ldots \ldots \ldots \ldots \ldots \ldots \ldots \ldots \ldots \ldots \ldots \ldots \ldots \ldots$

Table 1. Share of Trucks by Fuel Type and Truck Size -

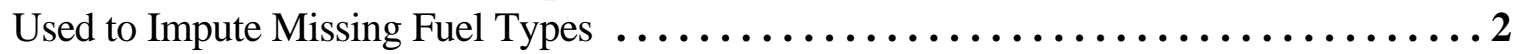

Table 2. Share of Trucks by Operator Class, Truck Size, and Fleet Indicator -

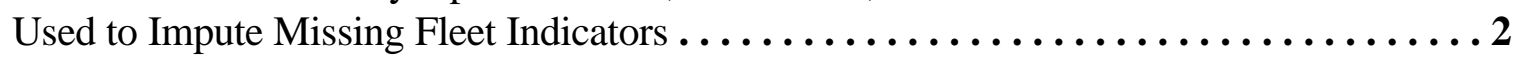

Table 3. Vius Principal Products Mapped to Nems Industrial Sectors . . . . . . . . . . 3

Table 4. Vius Principal Products Not Mapped to Nems Industrial Sectors . . . . . . . . . 4

Table 5. Shares of Vius Principal Products - Used to Reallocate

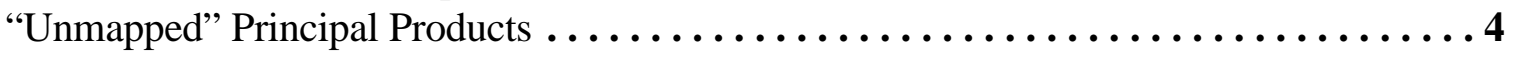

GOVERNMENT TRUCKS $\ldots \ldots \ldots \ldots \ldots \ldots \ldots \ldots \ldots \ldots \ldots \ldots \ldots \ldots \ldots . \ldots . \ldots \ldots$

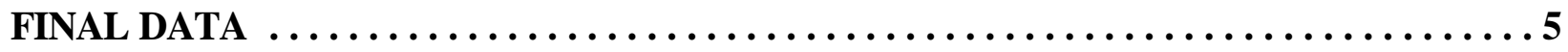

SUMMARY AND RECOMMENDATIONS $\ldots \ldots \ldots \ldots \ldots \ldots \ldots \ldots \ldots \ldots \ldots \ldots$

APPENDIX A. ................................... A-1

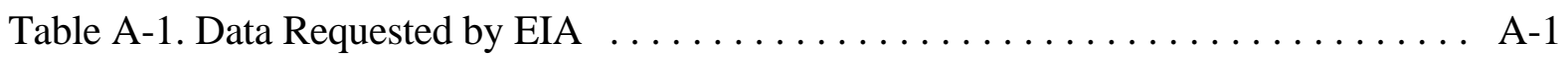

Table A-2. EIA Data Element Definitions $\ldots \ldots \ldots \ldots \ldots \ldots \ldots \ldots \ldots \ldots \ldots \ldots \ldots \ldots \ldots \ldots$

APPENDIX B. SAS PROGRAMS $\ldots \ldots \ldots \ldots \ldots \ldots \ldots \ldots \ldots \ldots \ldots \ldots \ldots$ B-1

APPENDIX C. HARDCOPY OF SPREADSHEET DELIVERED TO EIA ......... C-1

Updating the FTSAM: 1997 VIUS Data iii 



\begin{abstract}
The Energy Information Administration's (EIA's) National Energy Modeling System (NEMS) Freight Truck Stock Adjustment Model (FTSAM) was created in 1995 relying heavily on input data from the 1992 Economic Census, Truck Inventory and Use Survey (TIUS). The FTSAM is part of the NEMS Transportation Sector Model, which provides baseline energy projections and analyzes the impacts of various technology scenarios on consumption, efficiency, and carbon emissions. The base data for the FTSAM can be updated every five years as new Economic Census information is released.

Because of expertise in using the TIUS database, Oak Ridge National Laboratory (ORNL) was asked to assist the EIA when the new Economic Census data were available. ORNL provided the necessary base data from the 1997 Vehicle Inventory and Use Survey (VIUS) and other sources to update the FTSAM. ${ }^{1}$

The next Economic Census will be in the year 2002. When those data become available, the EIA will again want to update the FTSAM using the VIUS. This report, which details the methodology of estimating and extracting data from the 1997 VIUS Microdata File, should be used as a guide for generating the data from the next VIUS so that the new data will be as compatible as possible with the data in the model.
\end{abstract}

${ }^{1}$ The U.S. Census Bureau changed the survey name from Truck Inventory and Use Survey to Vehicle Inventory and Use Survey in 1997 in anticipation of including other vehicle types; however, the budget did not allow additional vehicle types to be included in the 1997 survey. 


\section{UPDATING THE FREIGHT TRUCK STOCK ADJUSTMENT MODEL: 1997 VEHICLE INVENTORY AND USE SURVEY DATA}

\section{INTRODUCTION}

The Energy Information Administration's (EIA's) National Energy Modeling System (NEMS) Freight Truck Stock Adjustment Model (FTSAM) was created in 1995 relying heavily on input data from the 1992 Economic Census, Truck Inventory and Use Survey (TIUS). The FTSAM is part of the NEMS Transportation Sector Model, which provides baseline energy projections and analyzes the impacts of various technology scenarios on consumption, efficiency, and carbon emissions. (Decision Analysis Corporation of Virginia, 1995, p. 1) The base data for the FTSAM can be updated every five years as new Economic Census information is released.

\section{OBJECTIVE}

Because of expertise in using the TIUS database, Oak Ridge National Laboratory (ORNL) was asked to assist the EIA when the new Economic Census data were available. ORNL provided the necessary base data from the 1997 Vehicle Inventory and Use Survey (VIUS) to update the FTSAM. $^{1}$ (U.S. Census Bureau, 2000) EIA provided a listing of 11 data tabulations necessary to update the FTSAM and a list of definitions for the data elements (See Appendix A). This report documents the procedure used for producing those data.

\section{VIUS DATA PREPARATION}

The VIUS database was transferred from its original MS-Access format to Statistical Analysis Software $\left(\mathrm{SAS}^{\odot}\right)$ format and the data were verified by comparing totals of trucks and truck-miles against the published VIUS report. (U.S. Census Bureau, 1999, Table 2a) Since the FTSAM is a freight truck model, the light trucks (less than 10,000 pounds gross vehicle weight) were excluded from the analysis on the basis that they are not freight trucks.

${ }^{1}$ The U.S. Census Bureau changed the survey name from Truck Inventory and Use Survey to Vehicle Inventory and Use Survey in 1997 in anticipation of including other vehicle types; however, the budget did not allow additional vehicle types to be included in the 1997 survey. 
Preliminary checks of the VIUS data indicated that most data elements required for this project were complete (i.e., non-missing). However, two important data elements had missing values. The fuel type was missing for $2 \%$ of all medium/heavy trucks and the fleet/non-fleet indicator was missing for $14 \%$ of all medium/heavy trucks. The missing data were imputed using the existing data proportions by size class (see Tables 1 and 2).

Table 1.

Share of Trucks by Fuel Type and Truck Size Used to Impute Missing Fuel Types

\begin{tabular}{|l|c|c|}
\hline Fuel type & Medium trucks & Heavy trucks \\
\hline Gasoline & $56.9 \%$ & $9.7 \%$ \\
\hline Diesel & $41.3 \%$ & $90.2 \%$ \\
\hline Liquified Petroleum Gas (LPG) $^{a}$ & $1.6 \%$ & $0.1 \%$ \\
\hline Other $^{a}$ & $0.2 \%$ & $0.1 \%$ \\
\hline Total $^{a}$ & $100.0 \%$ & $100.0 \%$ \\
\hline
\end{tabular}

${ }^{a}$ The "Other" fuel type was assumed to be compressed natural gas (CNG).

Table 2.

Share of Trucks by Operator Class, Truck Size, and Fleet Indicator Used to Impute Missing Fleet Indicators

\begin{tabular}{|l|c|c|c|c|}
\hline & \multicolumn{2}{|c|}{ Medium trucks } & \multicolumn{2}{c|}{ Heavy trucks } \\
\hline Operator class & Non-fleet & Fleet & Non-fleet & Fleet \\
\hline Business & $62.3 \%$ & $37.7 \%$ & $47.7 \%$ & $52.3 \%$ \\
\hline For-Hire & $21.9 \%$ & $78.2 \%$ & $29.3 \%$ & $70.7 \%$ \\
\hline Mixed & $100.0 \%$ & $0.0 \%$ & $97.0 \%$ & $3.0 \%$ \\
\hline
\end{tabular}

Note: It was assumed that all trucks with operator class equal to "personal" were $100 \%$ non-fleet vehicles and all trucks with operator class equal to "daily rental" were $100 \%$ fleet vehicles.

Next, the VIUS principal products (PRNPRO) were mapped into NEMS Industrial sectors as shown in Table 3. There were eleven VIUS principal products which could not be mapped directly into the sectors (see Table 4). Thus, following the original FTSAM methodology, these vehicles were allocated among NEMS sectors 1-10 using the existing data proportions (see Table 5). Using 
the VIUS principal product codes, one cannot determine whether the vehicle belongs to the utility sector; however, the VIUS major use code can identify which trucks belong in the utility sector. Therefore, any record having VIUS major use (MAJUSE) equal to "utility," regardless of principal product, was changed to NEMS sector 11 - Utility. (Decision Analysis Corporation of Virginia, 1995, p. A-4.)

Table 3.

VIUS Principal Products Mapped to NEMS Industrial Sectors

\begin{tabular}{|c|c|c|c|}
\hline \multicolumn{2}{|r|}{ VIUS Principal Product } & \multicolumn{2}{|r|}{ NEMS Industrial Sector } \\
\hline $\begin{array}{l}09 \\
11\end{array}$ & $\begin{array}{l}\text { Chemicals } \\
\text { Plastics and/or rubber }\end{array}$ & 1 & $\begin{array}{l}\text { Chemicals, rubber and } \\
\text { plastics }\end{array}$ \\
\hline 12 & Primary metal products & 2 & Primary metals \\
\hline 03 & Processed foods & 3 & Processed foods \\
\hline 08 & Paper products & 4 & Paper processing \\
\hline 10 & Petroleum & 5 & Petroleum products \\
\hline $\begin{array}{l}05 \\
26\end{array}$ & $\begin{array}{l}\text { Building materials } \\
\text { Glass products }\end{array}$ & 6 & $\begin{array}{l}\text { Stone, clay, glass, and } \\
\text { concrete }\end{array}$ \\
\hline $\begin{array}{l}13 \\
14 \\
15 \\
19\end{array}$ & $\begin{array}{l}\text { Fabricated metal products } \\
\text { Machinery } \\
\text { Transportation equipment } \\
\text { Craftsman's equipment }\end{array}$ & 7 & Metal durables \\
\hline $\begin{array}{l}17 \\
16 \\
07 \\
27 \\
18\end{array}$ & $\begin{array}{l}\text { Textile mill products } \\
\text { Furniture or hardware } \\
\text { Lumber and fabricated wood products } \\
\text { Miscellaneous manufacturing } \\
\text { Household goods }\end{array}$ & 8 & Other manufacturing \\
\hline $\begin{array}{l}01 \\
02 \\
30 \\
06\end{array}$ & $\begin{array}{l}\text { Farm products } \\
\text { Live animals } \\
\text { Animal feed } \\
\text { Logs and forest products }\end{array}$ & 9 & Agriculture \\
\hline 04 & Mining products & 10 & Mining \\
\hline- & Utility (If MAJUSE = 09) & 11 & Utility \\
\hline- & Not included in VIUS & 12 & Government \\
\hline
\end{tabular}


Table 4.

VIUS Principal Products Not Mapped to NEMS Industrial Sectors

\begin{tabular}{|l|l|l|l|l|}
\hline 28 & Industrial waste water & & 24 & Personal transportation \\
\hline 21 & Scrap, refuse or garbage & & 33 & Passengers \\
\hline 20 & Mixed cargos & & 23 & No load carried \\
\hline 32 & Recyclable products & & 25 & Not in use \\
\hline 29 & EPA hazardous waste & & 22 & Other \\
\hline 31 & Non-EPA hazardous waste & & & \\
\hline
\end{tabular}

Table 5.

Shares of VIUS Principal Products

Used to Reallocate "Unmapped" Principal Products

\begin{tabular}{|r|l|c|}
\hline & NEMS Industrial Sector & Shares \\
\hline 1 & Chemicals, rubber and plastics & $5.0 \%$ \\
\hline 2 & Primary metals & $2.4 \%$ \\
\hline 3 & Processed foods & $11.1 \%$ \\
\hline 4 & Paper processing & $2.5 \%$ \\
\hline 5 & Petroleum products & $4.1 \%$ \\
\hline 6 & Stone, clay, glass, and concrete & $18.3 \%$ \\
\hline 7 & Metal durables & $20.0 \%$ \\
\hline 8 & Other manufacturing & $13.6 \%$ \\
\hline 9 & Agriculture & $22.2 \%$ \\
\hline 10 & Mining & $0.9 \%$ \\
\hline
\end{tabular}

\section{GOVERNMENT TRUCKS}

Since the scope of the VIUS does not include government trucks, these data had to be added into the database. The total number of Federal plus State and Local Government trucks was obtained from the 1997 Highway Statistics. (U.S. Department of Transportation, 1998, Table MV-9) Total Government trucks were then split into size classes using percentages from the report Fleet Vehicles in the United States. (Miaou, et al., 1992, Table A-2) According to that report, $13.2 \%$ of all Government trucks are medium trucks and 3.5\% are heavy trucks. Once the 
medium and heavy government trucks were added into the database, their fuel types and ages were allocated in the same proportions as the VIUS vehicles. All Government trucks were classified as fleet vehicles.

\section{FINAL DATA}

Once the missing VIUS data were imputed, the NEMS Industrial sectors were set up, and the government trucks were included, the final data tabulations were generated from the database. The output was a large tab-delimited file containing all of the data. This file was then imported into a Lotus 123 spreadsheet file and formatted to the final product, a hardcopy of which is in Appendix C. A quality check was also performed on the data; any cell in a table which was based on a sample of 5 VIUS records or less was "blanked out" for quality purposes.

\section{SUMMARY AND RECOMMENDATIONS}

ORNL used SAS ${ }^{\odot}$ to estimate and extract data from the Census Bureau's 1997 VIUS Microdata File to assist EIA in updating the FTSAM. Other sources used in the estimation process were the Highway Statistics 1997 and Fleet Vehicles in the United States. The NEMS Transportation Sector Model: Freight Truck Stock Adjustment Model Update, which details the original creation of the FTSAM, was used as a guide to assist with compatibility between the 1992 FTSAM base data and 1997 data. The final data were delivered to EIA in spreadsheet format.

The next Economic Census will be in the year 2002. When those data become available, the EIA will again want to update the FTSAM using the VIUS. This report, which details the methodology of estimating and extracting data from the 1997 VIUS Microdata File, should be used as a guide for generating the data from the next VIUS so that the new data will be as compatible as possible with the data in the model. 



\section{REFERENCES}

Decision Analysis Corporation of Virginia, NEMS Transportation Sector Model: Freight Truck Stock Adjustment Model Update, Vienna, VA, November 1995, Appendix A.

Miaou, Shaw-Pin, Patricia S. Hu, and Jennifer R. Young, Oak Ridge National Laboratory, Fleet Vehicles in the United States: Composition, Operating Characteristics, and Fueling Practices, ORNL-6717, May 1992, Table A-2.

U.S. Census Bureau, 1997 Economic Census, Vehicle Inventory and Use Survey, United States, EC97TV-US, Washington, DC, October 1999, Table 2a.

U.S. Census Bureau, 1997 Vehicle Inventory and Use Survey, Microdata File, CD-EC97-VIUS, Washington, DC, January 2000.

U.S. Department of Transportation, Federal Highway Administration, Highway Statistics 1997, FHWA-PL-98-020, Washington, DC, December 1998, Tables MV-9 and VM-1. 



\section{APPENDIX A EIA REQUEST AND DEFINITIONS}

Table A-1.

Data Requested by EIA

\begin{tabular}{|c|c|c|c|c|c|c|c|}
\hline TABLE & EIA VARIABLE & DATA NEEDED & SECTOR & $\begin{array}{l}\text { SIZE } \\
\text { CLASS }\end{array}$ & $\begin{array}{l}\text { FUEL } \\
\text { TYPE }\end{array}$ & $\begin{array}{l}\text { FLEET/ } \\
\text { NON- } \\
\text { FLEET }\end{array}$ & AGE \\
\hline 1 & $\begin{array}{l}\text { Base year market share } \\
\text { of each fuel type }\end{array}$ & Number of NEW trucks & & $X$ & X & $X$ & \\
\hline 2 & \begin{tabular}{|l} 
Parameter representing \\
the tendency of each \\
sector to purchase diesel \\
trucks
\end{tabular} & \begin{tabular}{|l} 
Number of DIESEL \\
trucks
\end{tabular} & $X$ & $X$ & & $X$ & \\
\hline 3 & $\begin{array}{l}\text { Base year alternative fuel } \\
\text { share }\end{array}$ & $\begin{array}{l}\text { Number of NEW } \\
\text { ALTERNATIVE FUEL } \\
\text { trucks }\end{array}$ & $X$ & X & $X$ & $X$ & \\
\hline 4 & Base year truck stock & Number of trucks & $X$ & $X$ & $X$ & $X$ & $X$ \\
\hline 5 & Fleet transfer rates & $\begin{array}{l}\text { Number of FLEET } \\
\text { trucks }\end{array}$ & $X$ & $X$ & & $X$ & $X$ \\
\hline 6 & $\begin{array}{l}\text { Base year annual vehicle } \\
\text { miles traveled per truck }\end{array}$ & $\begin{array}{l}\text { Average annual vehicle } \\
\text { miles traveled per truck }\end{array}$ & $X$ & $X$ & $X$ & & $X$ \\
\hline 7 & \begin{tabular}{|l} 
Base year vehicle miles \\
traveled
\end{tabular} & $\begin{array}{l}\text { Total vehicle miles } \\
\text { traveled }\end{array}$ & $X$ & & & & \\
\hline 8 & \begin{tabular}{|l} 
Vehicle miles of travel \\
size class allocation \\
factor
\end{tabular} & $\begin{array}{l}\text { Total vehicle miles } \\
\text { traveled }\end{array}$ & $X$ & $X$ & & & \\
\hline 9 & $\begin{array}{l}\text { Fleet share of truck } \\
\text { purchases }\end{array}$ & $\begin{array}{l}\text { Number of NEW } \\
\text { FLEET trucks }\end{array}$ & $X$ & $X$ & & & \\
\hline 10 & $\begin{array}{l}\text { Truck share of miles in } \\
\text { urban areas }\end{array}$ & $\begin{array}{l}\text { Share of miles driven in } \\
\text { URBAN areas }{ }^{1}\end{array}$ & & $X$ & & & \\
\hline 11 & Base year fuel economy & \begin{tabular}{|l} 
Average fuel \\
economy
\end{tabular} & & $X$ & $X$ & & $X$ \\
\hline
\end{tabular}

${ }^{1}$ VIUS does not contain this information; Highway Statistics 1997 was used. (U.S. Department of Transportation, 1998, Table VM-1.)

${ }_{2}^{2}$ Medium trucks with fuel economy over 13 miles per gallon and heavy trucks over 11 miles per gallon were not used for this data estimation in order to be compatible with original model methodology. (Decision Analysis Research Corporation of Virginia, 1995, p. A-15.) 
Table A-2.

EIA Data Element Definitions

\begin{tabular}{|c|c|}
\hline Sector & $\begin{array}{l}\text { Chemicals, rubber and plastics } \\
\text { Primary metals } \\
\text { Processed foods } \\
\text { Paper processing } \\
\text { Petroleum products } \\
\text { Stone, clay, glass, and concrete } \\
\text { Metal durables } \\
\text { Other manufacturing } \\
\text { Agriculture } \\
\text { Mining } \\
\text { Utility } \\
\text { Government }\end{array}$ \\
\hline Size class & $\begin{array}{l}\text { Medium trucks ( } 10,000-26,000 \text { pounds gross vehicle weight) } \\
\text { Heavy trucks (greater than } 26,000 \text { pounds gross vehicle weight) }\end{array}$ \\
\hline Fuel type & $\begin{array}{l}\text { Diesel } \\
\text { Gasoline } \\
\text { Liquified Petroleum Gas } \\
\text { Compressed Natural Gas }\end{array}$ \\
\hline Fleet/Non-fleet & $\begin{array}{l}\text { Fleet (10 or more vehicles) } \\
\text { Non-fleet (less than } 10 \text { vehicles) }\end{array}$ \\
\hline Age & $\begin{array}{l}\text { New } \\
1 \text { year old } \\
2 \text { year old } \\
3 \text { year old } \\
4 \text { year old } \\
5 \text { year old } \\
6 \text { year old } \\
7 \text { year old } \\
8 \text { year old } \\
9 \text { year old } \\
10 \text { years old or older }\end{array}$ \\
\hline
\end{tabular}




\section{APPENDIX B SAS PROGRAMS}

These programs were run using PC-SAS Version 6.11 on a Dell Dimension XPS T550 personal computer. Program 1 generates data to use to impute missing data elements. Program 2 adds government trucks to the VIUS file, imputes the necessary data elements for government and nongovernment trucks, and creates the NEMS Industrial sector. Program 3 creates the tabulations requested by EIA. The table numbers in Program 3 match those on the table descriptions in Appendix A. Program 4 is exactly the same as Program 3 except the number of records is tabulated instead of the number of trucks. This was used to determine data which were not of acceptable quality. Any cell in a table which was based on 5 VIUS records or less was "blanked out" for quality purposes. (Government vehicle data were not "blanked out" on this basis, because the few government records which were in the database were created in Program 2 using adequate VIUS data.)

Please note that in Programs 3 and 4, the Proc Tabulate options "NOSEPS FORMCHAR='0900000000090009000000'X" indicate that the output should be tab-delimited instead of the usual Proc Tabulate separator. The tab-delimited output is easily imported into any spreadsheet software (e.g., Lotus 123, MS-Excel). 


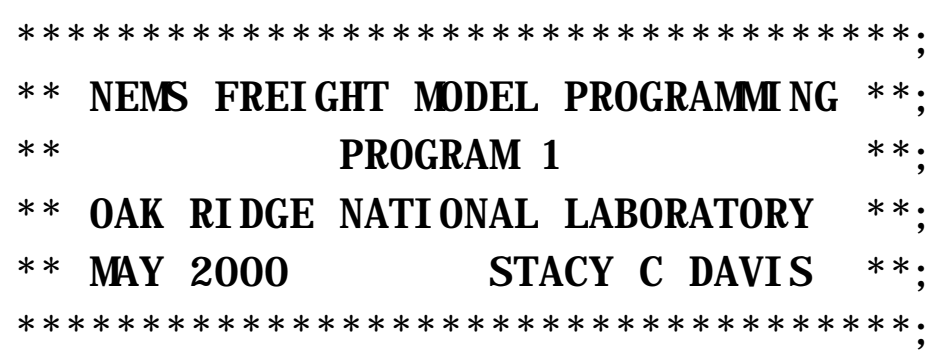

LI BNAME D ' D: \TI USI VI US97\ DATA' ;

** FORMAT SECTI ON **;

PROC FORMAT;

VALUE \$FUELTYP

' 1 ' = ' GASOLI NE'

' 2 ' = ' GASOLI NE'

' $3^{\prime}=$ ' DI ESEL'

' 4 ' = ' LPG'

' 5 ' $=$ ' CNG'

;

VALUE \$SI ZECLAS

' 1 ' = ' LI GHT'

' 2' = ' LI GHT'

' $3^{\prime}=$ ' MEDI UM

' 4 ' = ' MEDI UM

$' 5^{\prime}=$ ' MEDI UM

' 6 ' = ' MEDI UM

' 7 ' $=$ ' HEAVY'

' 8 ' = ' HEAVY'

;

VALUE \$FLSTAT

' 01 ' = 'NON- FLEET'

' 02 ' $=$ ' NON- FLEET'

' $03^{\prime}=$ ' NON- FLEET'

' 04 ' = 'FLEET'

' $05^{\prime}=$ ' FLEET'

' $06^{\prime}=$ ' FLEET'

' 07 ' = 'FLEET' 


$$
\begin{aligned}
& { }^{\prime} 08^{\prime}=\text { ' FLEET' } \\
& { }^{\prime} 09^{\prime}=\text { ' FLEET' } \\
& { }^{\prime} 10^{\prime}=\text { ' FLEET' }
\end{aligned}
$$

VALUE \$PROD

$$
\begin{aligned}
& \text { ' } 01 \text { ' = '09' } \\
& \text { '02' = '09' } \\
& \text { ' } 03^{\prime}={ }^{\prime} 03^{\prime} \\
& \text { '04' = '10' } \\
& \text { '05' = '06' } \\
& \text { '06' = '09' } \\
& \text { '07'= '08' } \\
& \text { '08' = '04' } \\
& \text { ' } 09^{\prime}={ }^{\prime} 01^{\prime} \\
& \text { ' } 10^{\prime}={ }^{\prime} 05^{\prime} \\
& \text { ' } 11^{\prime}={ }^{\prime} 01^{\prime} \\
& \text { ' } 12^{\prime}={ }^{\prime} 02^{\prime} \\
& \text { ' } 13^{\prime}={ }^{\prime} 07^{\prime} \\
& \text { ' } 14^{\prime}={ }^{\prime} 07^{\prime} \\
& \text { ' } 15^{\prime}={ }^{\prime} 07 \\
& \text { ' } 16^{\prime}={ }^{\prime} 08^{\prime} \\
& \text { ' } 17^{\prime}={ }^{\prime} 08^{\prime} \\
& \text { ' } 18^{\prime}={ }^{\prime} 08^{\prime} \\
& \text { ' } 19^{\prime}={ }^{\prime} 07^{\prime} \\
& \text { ' } 26^{\prime}={ }^{\prime} 06^{\prime} \\
& \text { ' } 27^{\prime}={ }^{\prime} 08^{\prime} \\
& \text { ' } 20^{\prime}={ }^{\prime} \text { ' 99' } \\
& \text { ' } 21^{\prime}={ }^{\prime} 99^{\prime} \\
& \text { ' } 22^{\prime}=\text { ' 99' } \\
& \text { ' } 23^{\prime}=\text { ' 99' } \\
& \text { ' } 24^{\prime}={ }^{\prime} \text { '99' } \\
& \text { ' } 25^{\prime}=\text { ' 99' } \\
& \text { ' } 28^{\prime}={ }^{\prime} \text { ' 99' } \\
& \text { ' 29' = ' 99' } \\
& \text { ' } 30^{\prime}={ }^{\prime} 09^{\prime} \\
& \text { ' } 31^{\prime}={ }^{\prime} 99^{\prime} \\
& \text { ' } 32^{\prime}={ }^{\prime} \text { ' 99' }
\end{aligned}
$$




$$
\begin{aligned}
& \text { ' } 33^{\prime}={ }^{\prime} 99^{\prime} \\
& \text {; }
\end{aligned}
$$

VALUE \$CLASS

' 1 ' = ' BUSI NESS'

' $3^{\prime}=$ ' FOR- HI RE'

' $5^{\prime}={ }^{\prime} M$ XED'

** SET UP THE DATABASE **;

TI TLE ' VI US97' ;

DATA VI US97; SET D. VI US97;

WHERE PKGWW NOT I N ( ' 1', ' 2' ); ** GET RI D OF THE LI GHT VEH CLES **;

SECTOR1 = PUT (PRNPRO, \$PROD. );

$* * * * * * * * * * * * * * * * * * * * * * * * * * * * * * * * * * * * * * * * * * * * * * * * * * * * * * * * * * * \pi ;$

** GET SHARES FOR USE I N I MPUTI NG M SSI NG VALUES **;

** AND SETTI NG UP THE PRODUCT SECTORS

************************************************************;

** SECTOR SHARES $* *$;

PROC TABULATE DATA $=V I$ US97;

WHERE SECTORI NE ' 99' ;

CLASS SECTOR1;

VAR EXPANF;

TABLES ( SECTOR $1 \rightleftharpoons$ NEMS SECTOR' ALL), EXPANF $\rightleftharpoons$ TRUCKS' $*$ PCTSUM* $F=6.2$;

TI TLE2 ' NON-M SSI NG SECTOR SHARES FOR USE I N ALLOCATI NG

12 PRNPRO THAT DO NOT GO ELSEWHERE' ;

** FUEL TYPE SHARES **;

PROC TABULATE DATA $=V I$ US97;

WHERE ENGTYP NE ' ' ;

CLASS PKGWW ENGTYP;

FORMAT PKGWW \$SI ZECLAS. ENGTYP \$FUELTYP. ;

VAR EXPANF;

TABLES ( ENGTYP $=$ FUEL TYPE' ALL), PKGMH ' 
*EXPANF $=$ TRUCKS ${ }^{*}$ PCTSUMKENGTYP ALL $>* F=6.2$;

TI TLE2 ' NON-M SSI NG FUEL TYPE SHARES FOR USE I N

I MPUTI NG M SSI NG VALUES' ;

** SHARES BY OPERATOR CLASS, TRUCK SI ZE, AND FLEET I NDI CATOR **;

PROC TABULATE DATA=VI US97;

WHERE FLTSZE NE ' ' AND OPCLAS I N (' 1', ' 3' , ' 5' );

CLASS PKGWW OPCLAS FLTSZE;

FORMAT PKGWW \$SI ZECLAS. FLTSZE \$FLSTAT. OPCLAS \$CLASS.;

VAR EXPANF;

TABLES OPCLAS, PKGW $=1 *($ FLTSZE $=1$ ALL $)$

*EXPANF $=$ TRUCKS' *PCTSUMKFLTSZE ALL $>* F=6.2$;

TI TLE2 ' NON-M SSI NG FLEET I NDI CATOR SHARES BY OPERATOR CLASS

AND SI ZE CLASS' ;

TI TLE3 ' FOR USE I N I MPUTI NG M SSI NG VALUES' ;

RUN;

** AGE SHARES FOR GOVERNMENT TRUCKS **;

PROC TABULATE DATA $=V I$ US97;

CLASS MDLYR PKGWW

FORMAT PKGWW \$SI ZECLAS. ;

VAR EXPANF;

TABLE MDLYR ALL, PKGW $=1 *$ EXPANF $=$ TRUCKS' *

PCTSUMKMDLYR ALL $>* F=6$. 2;

TI TLE2 ' AGE SHARES FOR USE I N I MPUTI NG AGE VALUES

FOR GOVERNMENT TRUCKS' ;

RUN; 


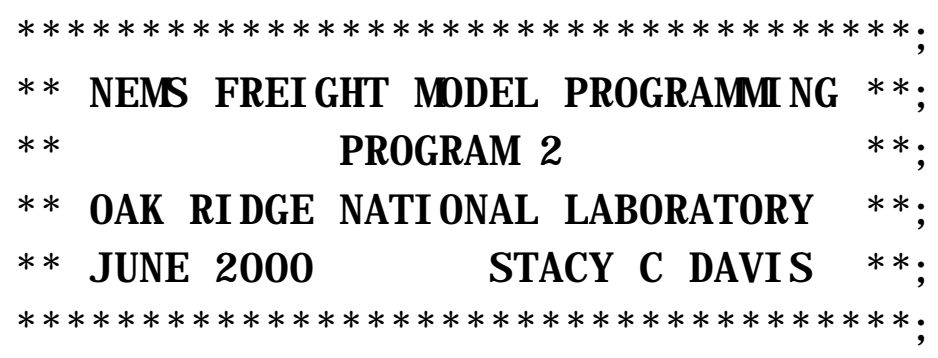

LI BNAME D ' D: \TI US\ VI US97\ DATA' ;

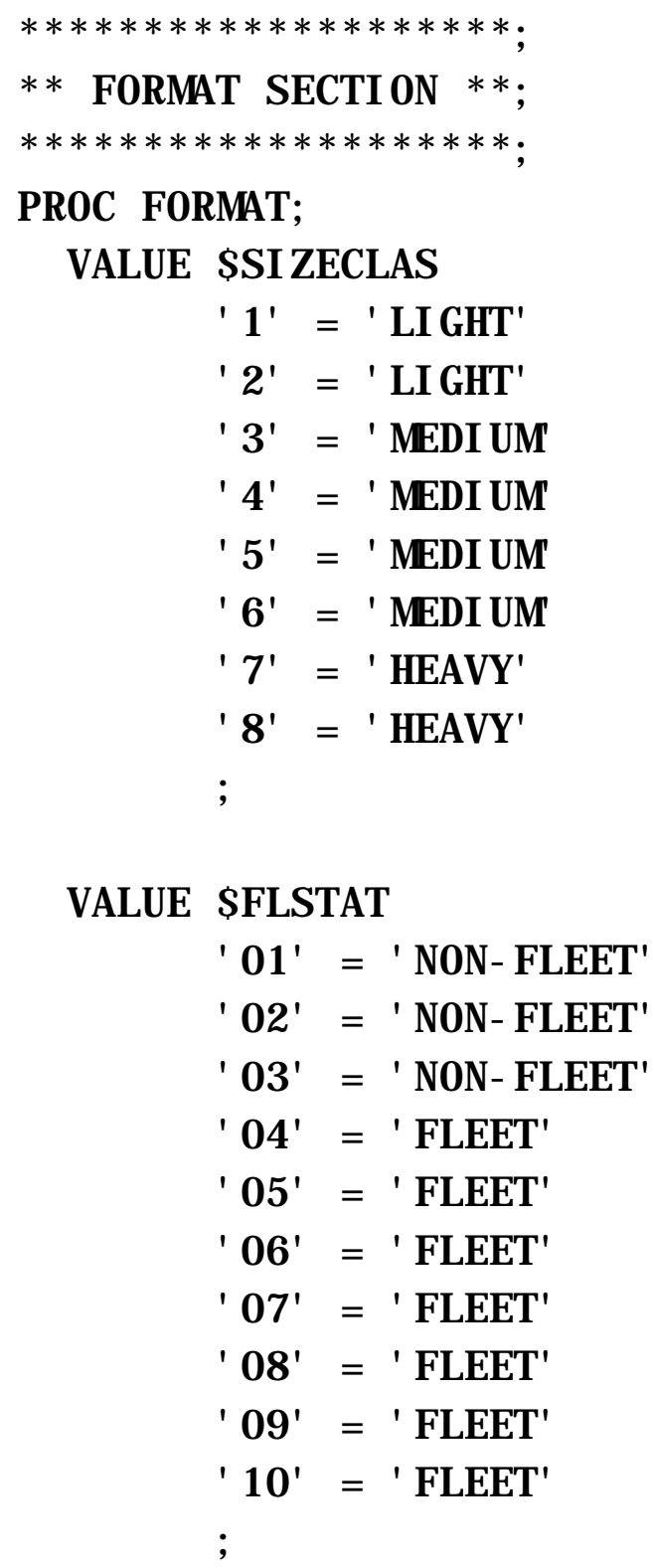


VALUE \$PROD

${ }^{\prime} 01^{\prime}={ }^{\prime} 09 '$

${ }^{\prime} 02^{\prime}={ }^{\prime} 09^{\prime}$

' $03^{\prime}={ }^{\prime} 03^{\prime}$

' $04^{\prime}={ }^{\prime} 10^{\prime}$

'05' = '06'

${ }^{\prime} 06^{\prime}={ }^{\prime} 09^{\prime}$

'07' = '08'

' $08^{\prime}={ }^{\prime} 04^{\prime}$

'09' = '01'

' $10^{\prime}={ }^{\prime} 05^{\prime}$

' 11 ' = '01'

' $12^{\prime}={ }^{\prime} 02^{\prime}$

' $13^{\prime}={ }^{\prime} 07^{\prime}$

' $14^{\prime}={ }^{\prime} 07^{\prime}$

' $15^{\prime}={ }^{\prime} 07^{\prime}$

' $16^{\prime}={ }^{\prime} 08^{\prime}$

' $17^{\prime}={ }^{\prime} 08^{\prime}$

' $18^{\prime}={ }^{\prime} 08^{\prime}$

' 19' = '07'

' 26' = '06'

' $27^{\prime}={ }^{\prime} 08^{\prime}$

' $20^{\prime}={ }^{\prime} 99^{\prime}$

' $21^{\prime}={ }^{\prime}$ ' 99'

' $22^{\prime}={ }^{\prime} 99^{\prime}$

' $23^{\prime}={ }^{\prime} 99^{\prime}$

' 24 ' = ' 99'

' $25^{\prime}={ }^{\prime} 99^{\prime}$

' $28^{\prime}=$ ' 99'

' $29^{\prime}=$ ' 99'

' $30^{\prime}={ }^{\prime} 09^{\prime}$

' $31^{\prime}=$ ' 99'

' $32^{\prime}=$ ' 99'

' $33^{\prime}=$ ' 99'

$' 77^{\prime}={ }^{\prime} 12^{\prime}$ 


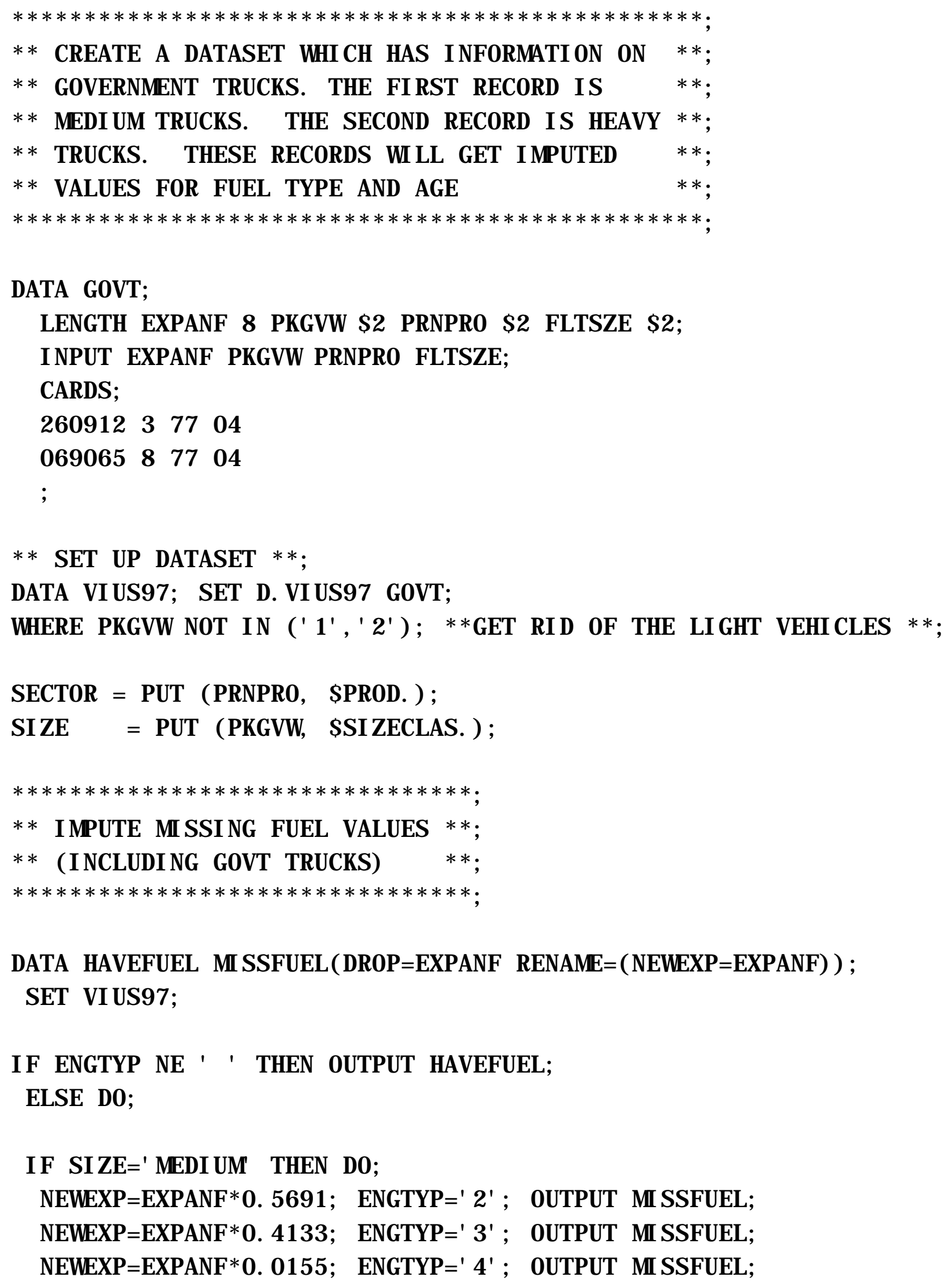

LENGTH EXPANF 8 PKGWW \$2 PRNPRO \$2 FLTSZE \$2;

I NPUT EXPANF PKGWW PRNPRO FLTSZE;

CARDS;

26091237704

06906587704

;

** SET UP DATASET **;

DATA VI US97; SET D. VI US97 GOVT;

WHERE PKGWW NOT I N ( ' 1', ' 2' ); **GET RI D OF THE LI GHT VEH CLES **;

SECTOR = PUT ( PRNPRO, \$PROD. $)$;

SI ZE = PUT (PKGWW \$SI ZECLAS. );

$* * * * * * * * * * * * * * * * * * * * * * * * * * * * * * * * ;$
$* *$ I MPUTE M SSI NG FUEL VALUES $* * ;$
$* *$ ( I NCLUDI NG GOVT TRUCKS) $* * ;$
$* * * * * * * * * * * * * * * * * * * * * * * * * * * * * * * * ;$

DATA HAVEFUEL M SSFUEL( DROP=EXPANF RENAME $=($ NEWEXP=EXPANF) );

SET VI US97;

I F ENGTYP NE ' ' THEN OUTPUT HAVEFUEL;

ELSE DO;

I F SI ZE $=$ MEDI UM THEN DO;

NEWEXP $=$ EXPANF*0. 5691; ENGTYP $=2$ ' ; OUTPUT M SSFUEL;

NEWEXP $=$ EXPANF* 0. 4133; ENGTYP $=31$; OUTPUT M SSFUEL;

NEWEXP=EXPANF*0. 0155; ENGTYP $=4$ ' ; OUTPUT M SSFUEL; 


\begin{abstract}
NEWEXP $=$ EXPANF $* 0.0021 ; \quad$ ENGTYP $=51$; OUTPUT M SSFUEL; END;
\end{abstract}

I F SI ZE = HEAVY' THEN DO;

NEWEXP $=$ EXPANF*0. 0967; ENGTYP $=2$ ' ; OUTPUT M SSFUEL; NEWEXP $=$ EXPANF*0. 9016; ENGTYP $=31$; OUTPUT M SSFUEL; NEVEXP $=$ EXPANF*0. 0010; ENGTYP $=44^{\prime}$; OUTPUT M SSFUEL; NEWEXP $=$ EXPANF* 0. 0007; ENGTYP $=51$; OUTPUT M SSFUEL; END;

END;

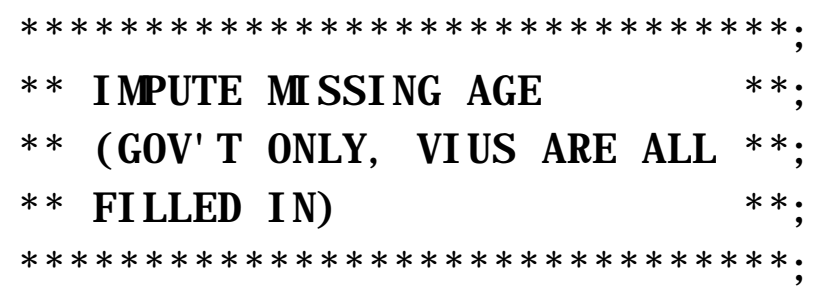

DATA HAVEAGE $M$ SSAGE( DROP=EXPANF RENAME $=($ NEWEXP=EXPANF) ); SET HAVEFUEL M SSFUEL;

I F MDLYR NE ' ' THEN OUTPUT HAVEAGE;

ELSE DO;

I F SI ZE $=$ MEDI UM THEN DO;

NEWEXP $=$ EXPANF*0. 0412; $M D L Y R=$ ' 01' ; OUTPUT M SSAGE;

NEWEXP $=E X P A N F * 0.0447 ; \quad M D L Y R=$ ' 02' ; OUTPUT M SSAGE;

NEWEXP $=E X P A N F * 0.0670 ; \quad M D L Y={ }^{*}$ 03' ; OUTPUT M SSAGE;

NEWEXP $=E X P A N F * 0.0476 ; \quad M D L Y R=$ ' 04' ; OUTPUT M SSAGE;

NEWEXP =EXPANF*0. 0376; $M D L Y R=$ ' 05' ; OUTPUT M SSAGE;

NEWEXP $=E X P A N F * 0.0303 ; \quad M D L Y={ }^{*} 06$ ' ; OUTPUT M SSAGE;

NEWEXP=EXPANF*0. 0385; $M D L Y R='$ 07' ; OUTPUT M SSAGE;

NEWEXP $=$ EXPANF*0. 0493; $M D L Y R=$ ' 08' ; OUTPUT M SSAGE;

NEWEXP $=$ EXPANF*0. 0525; $M D L Y R=$ ' 09' ; OUTPUT M SSAGE;

NEWEXP $=$ EXPANF*0. 0510; $M D L Y R=' 10$ ' ; OUTPUT M SSAGE;

NEWEXP=EXPANF*0. 5403; MDLYR= ' 11' ; OUTPUT M SSAGE;

END; 
I F SI ZE $=$ HEAVY' THEN DO;

NEWEXP=EXPANF*0. 0779; $M D L Y R=$ ' 01' ; OUTPUT M SSAGE;

NEWEXP=EXPANF*0. 0768; $M D L Y R={ }^{\prime} 02$ ' ; OUPUT M SSAGE;

NEWEXP=EXPANF*0. 0894; $M D L Y R=$ ' 03' ; OUTPUT M SSAGE;

NEWEXP=EXPANF*0. 0680; $M D L Y R=$ ' 04' ; OUTPUT M SSAGE;

NEWEXP=EXPANF*0. 0565; MDLYR= ' 05' ; OUPUT M SSAGE;

NEWEXP=EXPANF*0. 0414; $M D L Y R=$ ' 06' ; OUTPUT M SSAGE;

NEWEXP =EXPANF*0. 0380; $M D L Y R=$ ' 07' ; OUTPUT M SSAGE;

NEWEXP=EXPANF*0. 0473; $M D L Y R={ }^{\prime} 08$ ' ; OUTPUT M SSAGE;

NEWEXP=EXPANF*0. 0502; $M D L Y R={ }^{\prime} 09$ ' ; OUTPUT M SSAGE;

NEWEXP=EXPANF*0. 0486; $M D L Y R=' 10$; OUTPUT M SSAGE;

NEWEXP $=$ EXPANF*0. 4059; $M D L Y R=$ ' 11' ; OUTPUT M SSAGE; END;

END;

$* * * * * * * * * * * * * * * * * * * * * * * * * * * * * * * * * * * * * ;$

** I MPUTE M SSI NG FLEET I NDI CATORS $* *$;

***************************************;

DATA HAVEFLT M SSFLT( DROP=EXPANF RENAME=( NEWEXP=EXPANF) ); SET HAVEAGE M SSAGE;

I F FLTSZE NE ' ' THEN OUTPUT HAVEFLT;

ELSE DO;

I F OPCLAS $=2$ ' THEN DO;

NEWEXP=EXPANF; FLTSZE $=01^{\prime}$; OUTPUT M SSFLT;

END;

ELSE I $F \quad O P C L A S=4 '$ THEN DO;

NEWEXP=EXPANF; FLTSZE $=04$; OUTPUT M SSFLT;

END;

ELSE I $F$ OPCLAS $=1$ ' THEN DO;

I $F$ SI ZE $=$ MEDI UM THEN DO;

NEWEXP $=E X P A N F * 0.6226 ; \quad F L T S Z E=01$; OUTPUT M SSFLT;

NEWEXP =EXPANF*0. 3774; FLTSZE $=04$ ' ; OTPUT M SSFLT; 


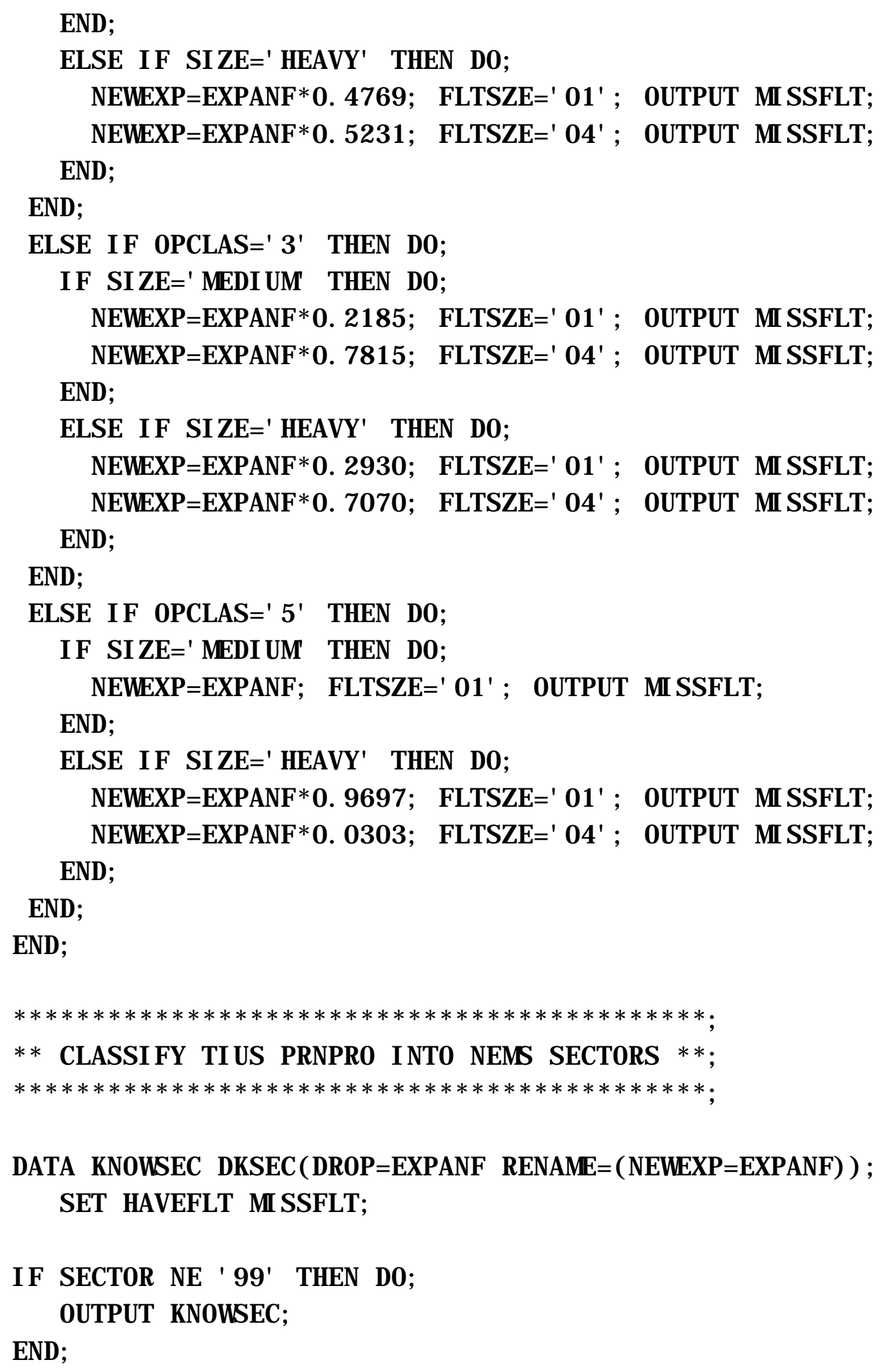

END;

ELSE I F SI ZE $=$ HEAVY' THEN DO;

NEWEXP $=E X P A N F * 0.4769 ; \quad F L T S Z E=01$ ' ; OUTPUT M SSFLT;

NEWEXP $=E X P A N F * 0.5231 ; \quad F L T S Z E=04{ }^{\prime}$; OUTPUT M SSFLT; END;

END;

ELSE I $F$ OPCLAS $=3^{\prime}$ THEN DO;

I $F$ SI ZE $=$ MEDI UM THEN DO;

NEWEXP $=$ EXPANF*0. 2185; FLTSZE $=01$ ' ; OUTPU M SSFLT;

NEWEXP $=E X P A N F * 0.7815 ; \quad F L T S Z E=04$ ' ; OUTPUT M SSFLT;

END;

ELSE I F SI ZE $=$ HEAY' THEN DO;

NEWEXP=EXPANF*0. 2930; FLTSZE $=01$ ' ; OUTPUT M SSFLT;

NEWEXP $=E X P A N F * 0.7070 ; \quad F L T S Z E=04{ }^{\prime}$; OUTPUT M SSFLT; END;

END;

ELSE I F OPCLAS $=5^{\prime}$ THEN DO;

I F SI ZE $=$ MEDI UM THEN DO;

NEWEXP=EXPANF; FLTSZE $=01^{\prime}$; OUTPUT M SSFLT;

END;

ELSE I F SI ZE $=$ HEAVY' THEN DO;

NEWEXP $=$ EXPANF*0. 9697; FLTSZE $=01$ ' ; OUTPUT M SSFLT;

NEWEXP $=$ EXPANF*0. 0303; FLTSZE $=04$; OUTPUT M SSFLT; END;

END;

END;

$* * * * * * * * * * * * * * * * * * * * * * * * * * * * * * * * * * * * * * * * * * * * * * * ;$

** CLASSI FY TI US PRNPRO I NTO NEMS SECTORS $* *$;

$* * * * * * * * * * * * * * * * * * * * * * * * * * * * * * * * * * * * * * * * * * * *$;

DATA KNOUSEC DKSEC( DROP=EXPANF RENAME $=($ NEWEXP $=E X P A N F))$; SET HAVEFLT M SSFLT;

I F SECTOR NE ' 99' THEN DO;

OUTPUT KNONSEC;

END; 


\section{ELSE DO;}

NEWEXP=EXPANF*0. 0500; SECTOR $=$ ' 01' ; OUTPUT DKSEC;

NEWEXP =EXPANF*0. 0236; SECTOR $=$ ' 02' ; OUTPUT DKSEC;

NEWEXP =EXPANF*0. 1111; SECTOR $=$ ' 03' ; OUTPUT DKSEC;

NEWEXP =EXPANF*0. 0248; SECTOR $=$ ' 04' ; OUTPUT DKSEC;

NEWEXP=EXPANF*0. 0410; SECTOR $=$ ' 05' ; OUTPUT DKSEC;

NEWEXP=EXPANF*0. 1826; SECTOR $=$ ' 06' ; OUTPUT DKSEC;

NEWEXP=EXPANF*0. 1996; SECTOR $=$ ' 07' ; OUTPUT DKSEC;

NEWEXP $=$ EXPANF*0. 1362; SECTOR $=$ ' 08' ; OUTPUT DKSEC;

NEWEXP=EXPANF*0. 2217; SECTOR $=$ ' 09' ; OUTPUT DKSEC;

NEWEXP=EXPANF*0. 0093; SECTOR $=$ ' 10' ; OUTPUT DKSEC; END;

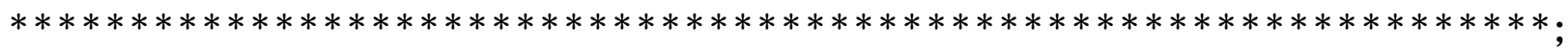

$* *$ SAVE PERMANENT DATASET WH CH NOW HAS NO M SSI NG FUEL TYPE OR**; $* *$ FLEET I NDI CATOR AND HAS THE EI A NEMS SECTORS PROPERLY CODED **; $* * * * * * * * * * * * * * * * * * * * * * * * * * * * * * * * * * * * * * * * * * * * * * * * * * * * * * * * * * * * * * * * *$;

DATA D. EI AVI U97; SET KNOUSEC DKSEC;

$* * * * * * * * * * * * * * * * * * * * * * * * * * * * * * * * * * * * * * * * * * * * * * * * * * * *$;

$* *$ CHANGE ALL RECORDS WTH MAJ OR USE $=$ UTI LI TY $* *$;

** TO THE UTI LI TY SECTOR

$* * * * * * * * * * * * * * * * * * * * * * * * * * * * * * * * * * * * * * * * * * * * * * * * * *$;

I $\mathrm{F}$ MAJ USE $=$ ' 09' THEN SECTOR $=$ ' 11 ' ;

RUN;

** TO CHECK THE TOTAL NUMBER OF TRUCKS **;

PROC TABULATE DATA=D. EI AVI U97 M SSI NG;

VAR EXPANF;

CLASS SI ZE;

TABLES SI ZE ALL, EXPANF*SUM*F $=15.2$;

TI TLE ' 1997 VI US' ;

TI TLE2 ' TOTAL TRUCKS ( VI US + GOVT) AFTER I MPUTATI ONS' ;

RUN; 


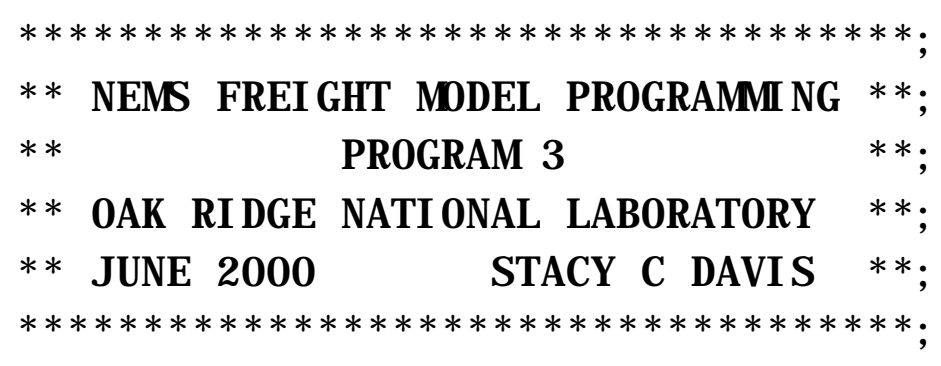

LI BNAME D ' D: \TI USI VI US97\ DATA' ;

OPTI ONS LI NESI ZE=110 NONUMBER NODATE;

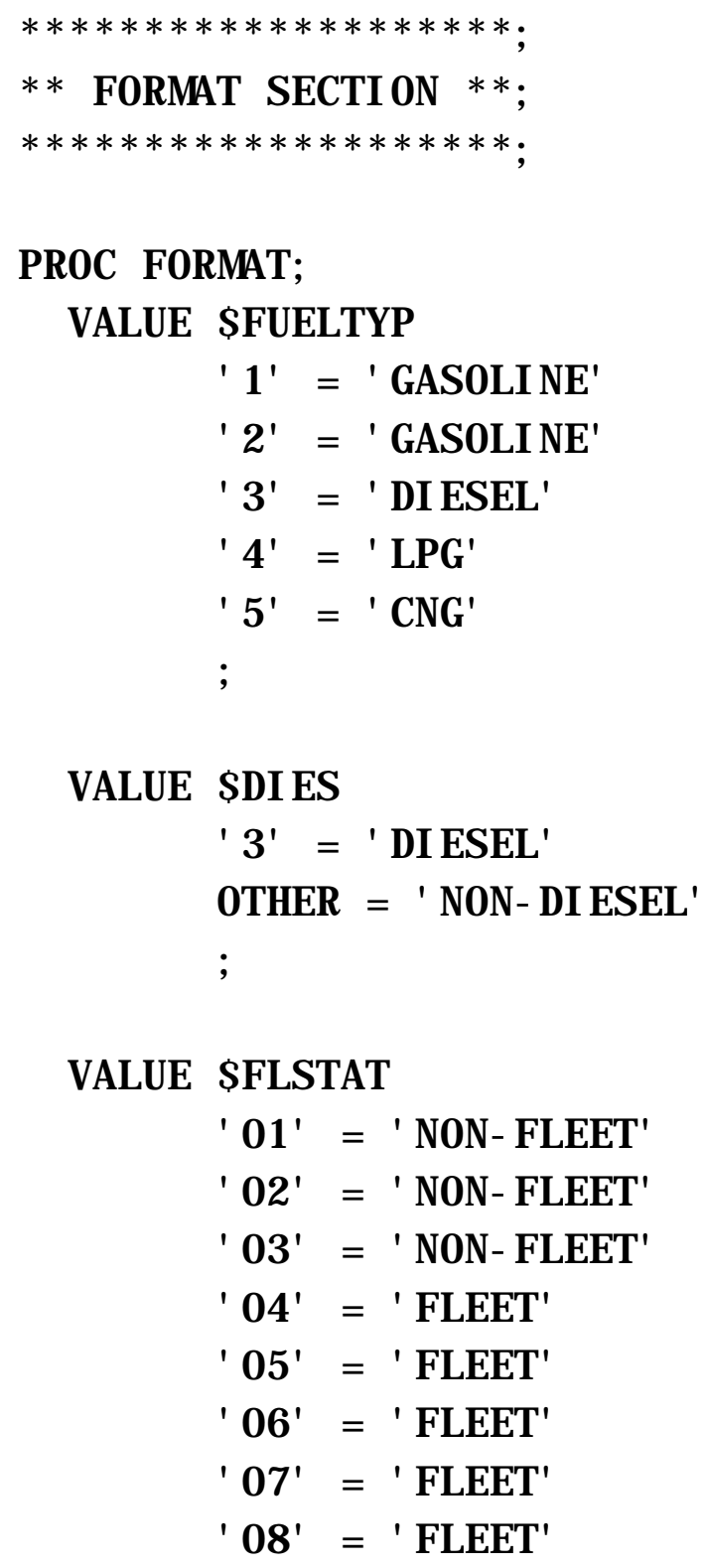




$$
\begin{aligned}
& { }^{\prime} 09^{\prime}=\text { ' FLEET' } \\
& { }^{\prime} 10^{\prime}=\text { ' FLEET' }
\end{aligned}
$$

VALUE \$USE

' 01 ' = ' CHEM CALS, RUBBER, PLASTI C'

' 02' = ' PRI MARY METALS'

' $03^{\prime}=$ ' PROCESSED FOODS'

' 04 ' = 'PAPER PRODUCTS'

' $05^{\prime}=$ ' PETROLEUM PRODUCTS'

' 06' = ' STONE, CLAY, GLASS, CONCRETE'

' 07' = ' METAL DURABLE'

' 08' = ' OTHER MANUFACTURI NG

' 09' = ' AGRI CULTURE'

$' 10^{\prime}=$ ' M NI NG'

' 11 ' = ' UTI LI TY'

' $12^{\prime}=$ ' GOVERNMENT'

;

VALUE \$AGE

' 01 ' = ' NEW

' 02 ' = ' 1 YR OLD'

'03' = ' 2 YR OLD'

' 04 ' = ' 3 YR OLD'

' $05^{\prime}=' 4$ YR OLD'

' $06^{\prime}=' 5$ YR OLD'

' 07 ' = ' 6 YR OLD'

$' 08 '=' 7$ YR OLD'

' 09' = ' 8 YR OLD'

' $10^{\prime}=$ ' 9 YR OLD'

' $11^{\prime}={ }^{\prime} 10+$ YR OLD'

TI TLE ' VI US 1997' ;

DATA EI AVI U97; SET D. EI AVI U97;

FLT=PUT( FLTSZE， \$FLSTAT. ) ;

I $\mathrm{F}$ ENGTYP $=$ ' 1 ' THEN ENGTYP $=$ ' 2 ' ; 
PROC SORT DATA=EI AVI U97; BY FLT SI ZE ENGTYP;

** TABLE $1 * *$;

PROC TABULATE DATA $=E I$ AVI U97 M SSI NG NOSEPS

$F O R M C H A R=0900000000090009000000^{\prime} X$;

WHERE MDLYR $=01^{\prime}$;

CLASS ENGTYP SI ZE;

FORMAT ENGTYP \$FUELTYP. ;

VAR EXPANF;

BY FLT;

LABEL FLT $=$ FLEET I NDI CATOR' ;

TABLES ( ENGTYP $\rightleftharpoons$ FUEL TYPE' $\quad A L L=$ TOTAL' $), \quad(S I Z E=\quad$ '

$\mathrm{ALL}=$ TOTAL' $) *$ EXPANF $=$ TRUCKS' $* \mathrm{SUM} \models \quad ' * \mathrm{~F}=$ COMMA10. ;

TI TLE2 ' NUMBER OF TRUCKS BY FUEL TYPE, SI ZE, AND FLEET STATUS' ;

RUN;

** TABLE 2**;

PROC TABULATE DATA=EI AVI U97 M SSI NG NOSEPS

FORMCHAR $=0900000000090009000000^{\prime} \mathrm{X}$;

WHERE MDLYR $=01^{\prime}$;

CLASS SECTOR SI ZE ENGTYP;

FORMAT SECTOR \$USE. ENGTYP \$DI ES. ;

VAR EXPANF;

BY FLT;

LABEL FLT $=$ FLEET I NDI CATOR' ;

TABLES SECTOR ALL $=$ TOTAL' , SI ZE $=1 *($ ENGTYP

$\mathrm{ALL}=$ TOTAL' $) *$ EXPANF $=$ TRUCKS' $* \mathrm{SUM} \models \quad$ '*F=COMMA10. ;

TI TLE2 ' NUMBER OF NEW TRUCKS BY SECTOR, SI ZE, AND FLEET STATUS' ;

RUN;

** TABLE $3 * *$;

PROC TABULATE DATA $=E I$ AVI U97 M SSI NG NOSEPS

FORMCHAR $=0900000000090009000000^{\prime} \mathrm{X}$;

WHERE MDLYR $\rightleftharpoons 01$ ';

CLASS SECTOR SI ZE ENGTYP;

FORMAT ENGTYP \$FUELTYP. SECTOR \$USE. ;

VAR EXPANF; 
BY FLT SI ZE;

LABEL FLT $=$ FLEET I NDI CATOR' ;

TABLES SECTOR ALL $=$ TOTAL' , $($ ENGTYP $=1) *$ EXPANF $=$ TRUCKS' $*$ SUM $\rightleftharpoons$ ' *F=COMMA10. ;

TI TLE2 ' NUMBER OF NEW TRUCKS BY SECTOR, SI ZE, FUEL TYPE AND FLEET STATUS' ;

RUN;

** SORT FOR TABLE 5**;

PROC SORT DATA=EI AVI U97 OUT=EI AVI U;

BY FLT SI ZE;

** TABLE $4 * *$;

PROC TABULATE DATA=EI AVI U97 M SSI NG NOSEPS

FORMCHAR $=0900000000090009000000^{\prime} X$;

CLASS SECTOR ENGTYP MDLYR;

FORMAT SECTOR \$USE. ENGTYP \$FUELTYP. MDLYR \$AGE.;

VAR EXPANF;

BY FLT SI ZE ENGTYP;

LABEL FLT $\rightleftharpoons$ FLEET I NDI CATOR' ;

TABLES MDLYR, SECTOR*EXPANF $=$ TRUCKS' *SUM $=1 * \mathrm{~F}=$ COMMA10. ;

TI TLE2 ' NUMBER OF TRUCKS BY FLEET I NDI CATOR, SI ZE, FUEL TYPE, SECTOR, AND AGE' ;

RUN;

$* *$ TABLE $5 * *$;

PROC TABULATE DATA=EI AVI U M SSI NG NOSEPS

FORMCHAR $=0900000000090009000000^{\prime} X$;

CLASS SECTOR MDLYR;

FORMAT SECTOR \$USE. ENGTYP \$FUELTYP. MDLYR \$AGE.;

VAR EXPANF;

BY FLT SI ZE;

LABEL FLT $\rightleftharpoons$ FLEET I NDI CATOR' ;

TABLES MDLYR, SECTOR*EXPANF $=$ TRUCKS' *SUM $\models ' *$ F=COMMA10. ;

TI TLE2 ' NUMBER OF TRUCKS BY FLEET I NDI CATOR, SI ZE, SECTOR, AND AGE' ;

TI TLE3 ' FOR FLEET SHARE OF TRUCK PURCHASES' ;

RUN; 
** SORT FOR TABLE $6 * *$;

PROC SORT DATA=EI AVI U97( KEEP=EXPANF ANNM L SI ZE ENGTYP SECTOR MDLYR) OUT=EI AVI U; BY SI ZE ENGTYP;

** TABLE $6 * *$;

PROC TABULATE DATA=D. EI AVI U97 M SSI NG NOSEPS

$F O R M C H A R=0900000000090009000000^{\prime} X$;

CLASS SECTOR SI ZE;

VAR ANNM L;

WEI GHT EXPANF;

TABLES SECTOR ALL $=$ TOTAL' , SI ZE $=1 * A N N M L=$ TOTAL VMT' *

$\mathrm{SUM} \models$ ' $* \mathrm{~F}=$ COMMA15. ;

TI TLE2 ' AVERAGE ANNUAL VMT PER TRUCK BY SECTOR \& SI ZE' ; RUN;

** TABLE $7 * *$;

PROC TABULATE DATA $=E I$ AVI U97 M SSI NG NOSEPS

FORMCHAR $=0900000000090009000000^{\prime} \mathrm{X}$;

CLASS SECTOR;

FORMAT SECTOR \$USE. ;

VAR ANNM L;

WEI GHT EXPANF;

TABLES SECTOR ALL $=$ TOTAL' , ANNM L $=$ TOTAL VMT' $*$ SUM $\models ~ ' * F=C O M M A 18$. ; TI TLE2 ' TOTAL VMT FOR 1997 BY SECTOR' ;

RUN;

** TABLES 8 AND $9 * *$;

PROC TABULATE DATA=EI AVI U97 M SSI NG NOSEPS

FORMCHAR $=0900000000090009000000^{\prime} X$;

WHERE MDLYR $=01^{\prime}$;

CLASS SECTOR SI ZE;

FORMAT SECTOR \$USE. ;

VAR EXPANF;

BY FLT;

TABLES SECTOR ALL $=$ TOTAL' , $($ SI ZE $A L L=$ TOTAL' $)$

*EXPANF $=$ TRUCKS' *SUM $=$ '*F=COMMA10. ;

TI TLE2 'NEW TRUCKS BY FLEET I NDI CATOR, SECTOR, AND SI ZE' ; 
RUN;

** PREPARE DATA FOR TABLE $11 * *$;

DATA EI AVI U; SET EI AVI U97( KEEP=MDLYR SI ZE ENGTYP MPG EXPANF);

I $F$ SI ZE = ' MEDI UM AND MPG GT 13 THEN DELETE;

I $\mathrm{F}$ SI ZE = ' HEAVY' AND MPG GT 11 THEN DELETE;

** SORT FOR TABLE $11 * *$;

PROC SORT DATA=EI AVI U;

BY SI ZE;

** TABLE $11 * *$;

PROC TABULATE DATA=EI AVI U M SSI NG NOSEPS

FORMCHAR $=0900000000090009000000^{\prime} X$;

CLASS MDLYR SI ZE ENGTYP;

FORMAT MDLYR \$AGE. ENGTYP \$FUELTYP. ;

VAR MPG;

WEI GHT EXPANF;

BY SI ZE;

TABLES MDLYR $=$ AGE' $A L L=$ TOTAL' , ENGTYP $=$ FUEL TYPE'

$* M P G^{*} M E A N=$ AVERAGE' *F $=10.5$;

TI TLE2 ' FUEL ECONOMY BY SI ZE, FUEL TYPE AND AGE' ; RUN; 


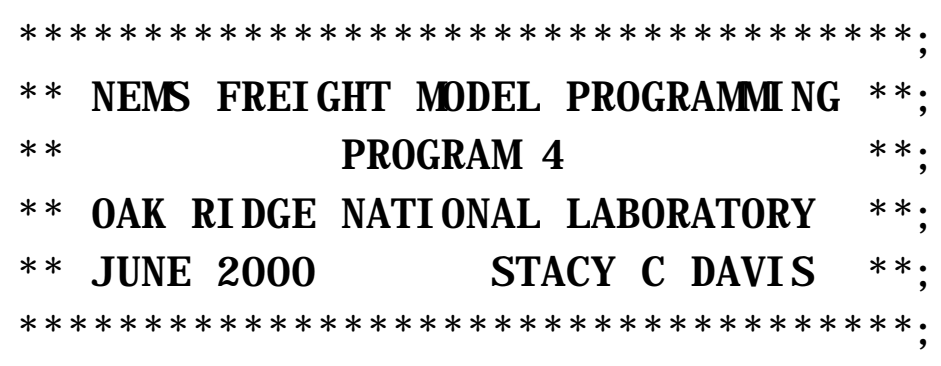

LI BNAME D ' D: \TI USI VI US97\ DATA' ;

OPTI ONS LI NESI ZE=110;

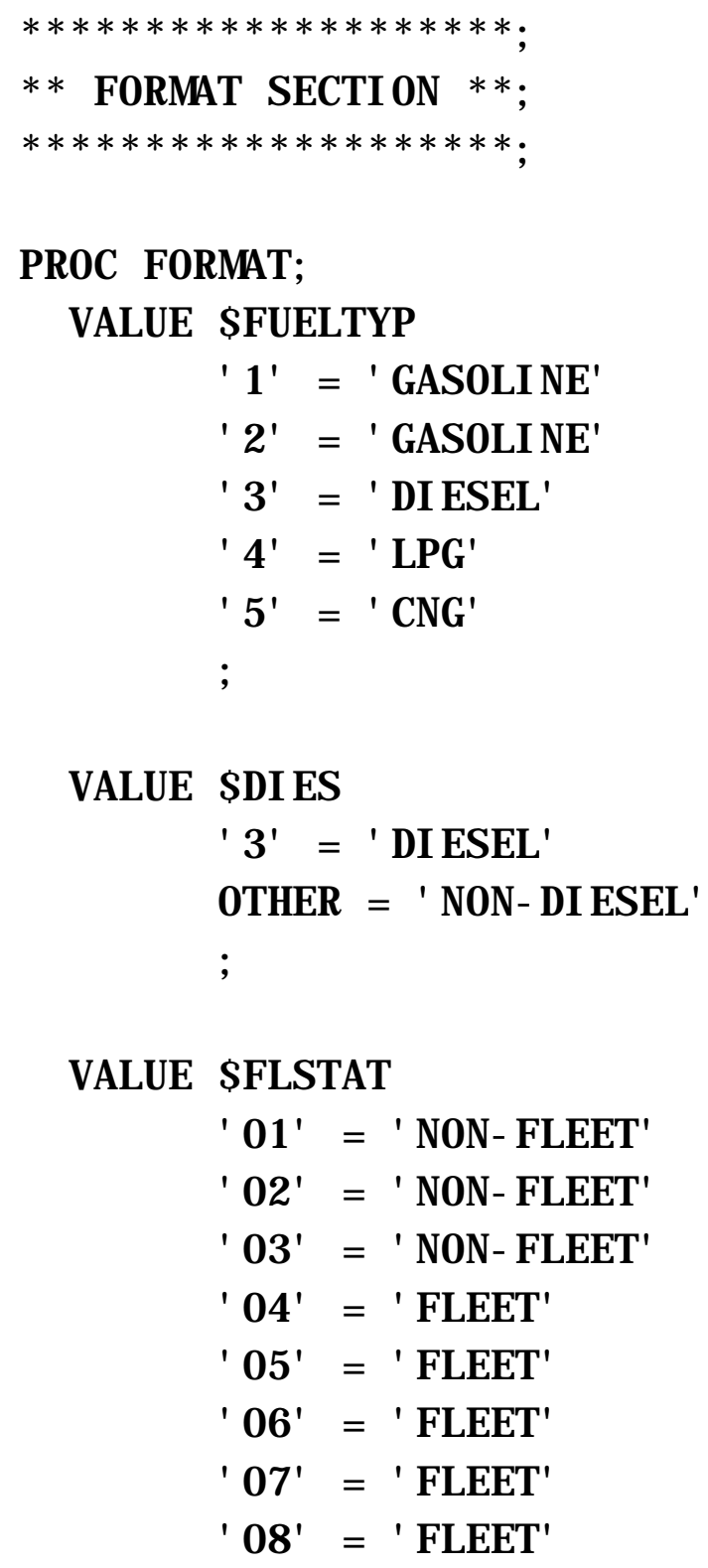




$$
\begin{aligned}
& { }^{\prime} 09^{\prime}=\text { ' FLEET' } \\
& { }^{\prime} 10^{\prime}=\text { ' FLEET' }
\end{aligned}
$$

VALUE \$USE

' 01 ' = ' CHEM CALS, RUBBER, PLASTI C'

' 02' = ' PRI MARY METALS'

' $03^{\prime}=$ ' PROCESSED FOODS'

' 04 ' = 'PAPER PRODUCTS'

' $05^{\prime}=$ ' PETROLEUM PRODUCTS'

' 06' = ' STONE, CLAY, GLASS, CONCRETE'

' 07' = ' METAL DURABLE'

' 08' = ' OTHER MANUFACTURI NG

' 09' = ' AGRI CULTURE'

$' 10^{\prime}=$ ' M NI NG'

' 11 ' = ' UTI LI TY'

' $12^{\prime}=$ ' GOVERNMENT'

;

VALUE \$AGE

' 01 ' = 'NEW

' 02 ' = ' 1 YR OLD'

'03' = ' 2 YR OLD'

' 04 ' = ' 3 YR OLD'

' $05^{\prime}=' 4$ YR OLD'

$' 06 '=' 5$ YR OLD'

' 07 ' = ' 6 YR OLD'

$' 08 '=' 7$ YR OLD'

' 09' = ' 8 YR OLD'

' $10^{\prime}=$ ' 9 YR OLD'

' $11^{\prime}={ }^{\prime} 10+$ YR OLD'

TI TLE ' VI US 1997' ;

DATA El AVI U97; SET D. El AVI U97;

FLT=PUT( FLTSZE， \$FLSTAT. ) ;

I $\mathrm{F}$ ENGTYP $=$ ' 1 ' THEN ENGTYP $=$ ' 2 ' ; 
PROC SORT DATA=EI AVI U97; BY FLT SI ZE ENGTYP;

** TABLE 1 **;

PROC TABULATE DATA=EI AVI U97 M SSI NG;

WHERE MDLYR $=01^{\prime}$;

CLASS ENGTYP SI ZE;

FORMAT ENGTYP \$FUELTYP. ;

VAR EXPANF;

BY FLT;

LABEL FLT $=$ FLEET I NDI CATOR' ;

TABLES ( ENGTYP $=$ FUEL TYPE' $\quad A L L=$ TOTAL' $), \quad(S I Z E=\quad$ '

$\mathrm{ALL}=\mathrm{TOTAL}$ ' $) * \mathrm{EXPANF}=$ TRUCKS' $* \mathrm{~N}^{*} \mathrm{~F}=$ COMMA10. ;

TI TLE2 ' NUMBER OF TRUCKS BY FUEL TYPE, SI ZE, AND FLEET STATUS' ;

RUN;

** TABLE 2**;

PROC TABULATE DATA=EI AVI U97 M SSI NG;

WHERE MDLYR $=01^{\prime}$;

CLASS SECTOR SI ZE ENGTYP;

FORMAT SECTOR \$USE. ENGTYP \$DI ES. ;

VAR EXPANF;

BY FLT;

LABEL FLT $=$ FLEET I NDI CATOR' ;

TABLES SECTOR ALL $=$ TOTAL' , SI ZE $=1 *($ ENGTYP

$\mathrm{ALL}=$ TOTAL' ) $*$ EXPANF $=$ TRUCKS' $* \mathrm{~N}^{*} \mathrm{~F}=$ COMMA10. ;

TI TLE2 ' NUMBER OF NEW TRUCKS BY SECTOR, SI ZE, AND FLEET STATUS' ;

RUN;

** TABLE $3 * *$;

PROC TABULATE DATA=EI AVI U97 M SSI NG;

WHERE $M D Y R=01^{\prime}$;

CLASS SECTOR SI ZE ENGTYP;

FORMAT ENGTYP \$FUELTYP. SECTOR \$USE. ;

VAR EXPANF;

BY FLT SI ZE;

LABEL FLT $=$ FLEET I NDI CATOR' ;

TABLES SECTOR ALL $=$ TOTAL' , (ENGTYP $=$ ' )

$*$ EXPANF $=$ TRUCKS ${ }^{*} N^{*} \mathrm{~F}=$ COMMA10. ; 
TI TLE2 ' NUMBER OF NEW TRUCKS BY SECTOR, SI ZE, FUEL TYPE AND FLEET STATUS' ;

RUN;

** TABLE $4 * *$;

PROC TABULATE DATA $=E I$ AVI U97 M SSI NG NOSEPS

FORMCHAR $=0900000000090009000000^{\prime} \mathrm{X}$;

CLASS SECTOR ENGTYP MDLYR;

FORMAT SECTOR \$USE. ENGTYP \$FUELTYP. MDLYR \$AGE.;

VAR EXPANF;

BY FLT SI ZE ENGTYP;

LABEL FLT $=$ FLEET I NDI CATOR' ;

TABLES MDLYR, SECTOR*EXPANF $=$ TRUCKS $* N * F=C O M M 10$. ;

TI TLE2 ' NUMBER OF TRUCKS BY FLEET I NDI CATOR, SI ZE, FUEL TYPE, SECTOR, AND AGE' ;

RUN;

** SORT FOR TABLE 5**;

PROC SORT DATA=EI AVI U97 OUT=EI AVI U;

BY FLT SI ZE;

** TABLE 5 **;

PROC TABULATE DATA=EI AVI U M SSI NG NOSEPS

FORMCHAR $=0900000000090009000000^{\prime} X$;

CLASS SECTOR MDLYR;

FORMAT SECTOR \$USE. ENGTYP \$FUELTYP. MDLYR \$AGE.;

VAR EXPANF;

BY FLT SI ZE;

LABEL FLT $=$ FLEET I NDI CATOR' ;

TABLES MDLYR, SECTOR*EXPANF $=$ TRUCKS $* N^{*} F=C O M M 10$. ;

TI TLE2 ' NUMBER OF TRUCKS BY FLEET I NDI CATOR, SI ZE, SECTOR, AND AGE' ;

TI TLE3 ' FOR FLEET SHARE OF TRUCK PURCHASES' ;

RUN;

** SORT FOR TABLE $5 * *$;

PROC SORT DATA=EI AVI U97(KEEP=EXPANF FLT SI ZE MDLYR) OUT=EI AVI U; BY SI ZE;

** TABLE $5 * *$; 
PROC TABULATE DATA=EI AVI U M SSI NG;

CLASS FLT MDLYR;

FORMAT MDLYR \$AGE. ;

VAR EXPANF;

BY SI ZE;

TABLES ( MDLYR $=$ AGE' $A L L=$ TOTAL' $), \quad(F L T$

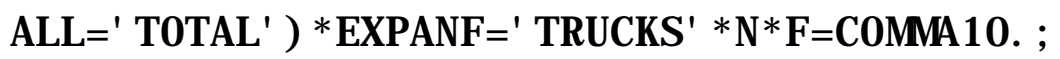

LABEL FLT $=$ FLEET I NDI CATOR' ;

TI TLE2 ' TRUCKS BY FLEET I NDI CATOR AND SI ZE CLASS' ;

RUN;

** SORT FOR TABLE $6 * *$;

PROC SORT DATA=EI AVI U97( KEEP=EXPANF ANNM L SI ZE ENGTYP SECTOR

MDLYR) OUT=EI AVI U;

BY SI ZE ENGTYP;

** TABLE $6 * *$;

PROC TABULATE DATA=D. EI AVI U97 M SSI NG NOSEPS

FORMCHAR $=0900000000090009000000^{\prime} \mathrm{X}$;

CLASS SECTOR SI ZE;

VAR ANNM L;

WEI GHT EXPANF;

TABLES SECTOR ALL $=$ TOTAL' , SI ZE $=1 * A N N M L=$ TOTAL VMT' *

$\mathrm{N}^{*} \mathrm{~F}=$ COMMA15. ;

TI TLE2 ' AVERAGE ANNUAL VMT PER TRUCK BY SECTOR \& SI ZE' ;

RUN;

** TABLE $7 * *$;

PROC TABULATE DATA=EI AVI U97 M SSI NG;

CLASS SECTOR;

FORMAT SECTOR \$USE. ;

VAR ANNM L;

WEI GHT EXPANF;

TABLES SECTOR ALL $=$ TOTAL' , ANNM $L=$ TOTAL VMT' $* N^{*} F=C O M M A 18$. ;

TI TLE2 ' TOTAL VMT FOR 1997 BY SECTOR' ;

RUN;

** TABLES 8 AND $9 * *$;

PROC TABULATE DATA=EI AVI U97 M SSI NG; 
WHERE MDLYR $=01^{\prime}$;

CLASS SECTOR SI ZE;

FORMAT SECTOR \$USE. ;

VAR EXPANF;

BY FLT;

TABLES SECTOR ALL $=$ TOTAL' , ( SI ZE

$A L L=$ TOTAL' ) $*$ EXPANF $=$ TRUCKS' $* N^{*} F=C O M M A 10$. ;

TI TLE2 ' NEW TRUCKS BY FLEET I NDI CATOR, SECTOR, AND SI ZE' ;

RUN;

** PREPARE DATA FOR TABLE $11 * *$;

DATA EI AVI U; SET EI AVI U97( KEEP=MDLYR SI ZE ENGTYP MPG EXPANF);

I $\mathrm{F}$ SI ZE = ' MEDI UM AND MPG GT 13 THEN DELETE;

I $F$ SI ZE = ' HEAV'' AND MPG GT 11 THEN DELETE;

** SORT FOR TABLE $11 * *$;

PROC SORT DATA=EI AVI U;

BY SI ZE;

** TABLE $11 * *$;

PROC TABULATE DATA=EI AVI U M SSI NG NOSEPS

$F O R M C H A R=0900000000090009000000^{\prime} \mathrm{X}$;

CLASS MDLYR SI ZE ENGTYP;

FORMAT MDLYR \$AGE. ENGTYP \$FUELTYP. ;

VAR MPG;

WEI GHT EXPANF;

BY SI ZE;

TABLES MDLYR $=$ AGE' $\quad A L L=$ TOTAL' , ENGTYP $=$ FUEL TYPE' $* M P G^{*} N^{*} F=10.5$;

TI TLE2 ' FUEL ECONOMY BY SI ZE, FUEL TYPE AND AGE' ;

RUN; 


\section{APPENDIX C}

\section{HARDCOPY OF SPREADSHEET DELIVERED TO EIA}


VIUS 1997

NUMBER OF NEW TRUCKS BY FUEL TYPE, SIZE, AND FLEET STATUS

FLEET INDICATOR=FLEET

\begin{tabular}{lrrrrrr}
\hline & HEAVY & MEDIUM & TOTAL & HEAVY & MEDIUM & TOTAL \\
FUEL TYPE & TRUCKS & TRUCKS & TRUCKS & TRUCKS & TRUCKS & TRUCKS \\
\hline GASOLINE & 1,731 & 21,914 & 23,645 & 2,940 & 20,268 & 23,208 \\
DIESEL & 152,101 & 54,352 & 206,454 & 36,917 & 26,973 & 63,890 \\
LPG & 5 & 680 & 685 & 0 & 295 & 295 \\
CNG & 4 & 413 & 416 & 48 & 1 & 49 \\
\hline TOTAL & 153,842 & 77,359 & 231,201 & 39,905 & 47,538 & 87,443 \\
\hline
\end{tabular}

FLEET INDICATOR=NON-FLEET 
VIUS 1997

NUMBER OF NEW TRUCKS BY SECTOR, SIZE, AND FLEET STATUS

FLEET INDICATOR=FLEET

SIZE=HEAVY

SIZE=MEDIUM

\begin{tabular}{lcrrrrrr}
\hline SECTOR & SECTOR \# & DIESEL & TOTAL & \% DIESEL & DIESEL & TOTAL & DIESEL \\
\hline CHEMICALS,RUBBER,PLASTIC & 01 & 9,375 & 9,395 & $99.79 \%$ & 2,932 & 3,251 & $90.19 \%$ \\
PRIMARY METALS & 02 & 4,813 & 4,821 & $99.83 \%$ & 471 & 566 & $83.22 \%$ \\
PROCESSED FOODS & 03 & 33,865 & 33,959 & $99.72 \%$ & 10,081 & 11,286 & $89.32 \%$ \\
PAPER PRODUCTS & 04 & 7,653 & 7,661 & $99.90 \%$ & 4,264 & 5,440 & $78.38 \%$ \\
PETROLEUM PRODUCTS & 05 & 4,714 & 4,729 & $99.68 \%$ & 1,350 & 2,252 & $59.95 \%$ \\
STONE,CLAY,GLASS,CONCRETE & 06 & 21,480 & 21,543 & $99.71 \%$ & 4,826 & 7,102 & $67.95 \%$ \\
METAL DURABLE & 07 & 17,775 & 18,102 & $98.19 \%$ & 7,411 & 10,399 & $71.27 \%$ \\
OTHER MANUFACTURING & 08 & 27,162 & 27,693 & $98.08 \%$ & 9,962 & 14,312 & $69.61 \%$ \\
AGRICULTURE & 09 & 17,394 & 17,500 & $99.39 \%$ & 3,798 & 5,236 & $72.54 \%$ \\
MINING & 10 & 2,449 & 2,453 & $99.84 \%$ & 102 & 140 & $72.86 \%$ \\
UTILITY & 11 & 569 & 606 & $93.89 \%$ & 4,713 & 6,624 & $71.15 \%$ \\
GOVERNMENT & 12 & 4,851 & 5,380 & $90.17 \%$ & 4,443 & 10,750 & $41.33 \%$ \\
\hline TOTAL & 152,101 & 153,842 & $98.87 \%$ & 54,352 & 77,359 & $70.26 \%$ \\
\hline
\end{tabular}

FLEET INDICATOR=NON-FLEET

SIZE=HEAVY

SIZE=MEDIUM

\begin{tabular}{|c|c|c|c|c|c|c|c|}
\hline SECTOR & SECTOR \# & DIESEL & TOTAL & $\%$ DIESEL & DIESEL & TOTAL & $\%$ DIESEL \\
\hline CHEMICALS,RUBBER,PLASTIC & 01 & 1,395 & 1,512 & $92.26 \%$ & 2,430 & 2,858 & $85.02 \%$ \\
\hline PRIMARY METALS & 02 & 1,079 & 1,112 & $97.03 \%$ & 451 & 541 & $83.36 \%$ \\
\hline PROCESSED FOODS & 03 & 4,725 & 5,322 & $88.78 \%$ & 1,597 & 3,431 & $46.55 \%$ \\
\hline PAPER PRODUCTS & 04 & 1,458 & 1,494 & $97.59 \%$ & 1,015 & 1,471 & $69.00 \%$ \\
\hline PETROLEUM PRODUCTS & 05 & 1,536 & 1,593 & $96.42 \%$ & 689 & 1,076 & $64.03 \%$ \\
\hline STONE,CLAY,GLASS,CONCRETE & 06 & 7,977 & 8,429 & $94.64 \%$ & 4,424 & 7,886 & $56.10 \%$ \\
\hline METAL DURABLE & 07 & 4,944 & 5,870 & $84.22 \%$ & 9,201 & 14,444 & $63.70 \%$ \\
\hline OTHER MANUFACTURING & 08 & 4,588 & 4,967 & $92.37 \%$ & 3,996 & 9,627 & $41.51 \%$ \\
\hline AGRICULTURE & 09 & 8,142 & 8,521 & $95.55 \%$ & 2,062 & 4,505 & $45.77 \%$ \\
\hline MINING & 10 & 900 & 913 & $98.58 \%$ & 218 & 254 & $85.83 \%$ \\
\hline UTILITY & 11 & 172 & 172 & $100.00 \%$ & 891 & 1,445 & $61.66 \%$ \\
\hline GOVERNMENT & 12 & & & & & & \\
\hline TOTAL & & 36,917 & 39,905 & $92.51 \%$ & 26,973 & 47,538 & $56.74 \%$ \\
\hline
\end{tabular}


VIUS 1997

NUMBER OF NEW TRUCKS BY SECTOR, SIZE, FUEL TYPE AND FLEET STATUS

FLEET INDICATOR=FLEET, SIZE=HEAVY

FLEET INDICATOR=FLEET, SIZE=MEDIUM

\begin{tabular}{|c|c|c|c|c|c|c|c|c|c|}
\hline SECTOR & SECTOR \# & GASOLINE & DIESEL & LPG & $\mathrm{CNG}$ & GASOLINE & $\overline{\text { DIESEL }}$ & $\overline{\mathrm{LPG}}$ & $\mathrm{CNG}$ \\
\hline CHEMICALS,RUBBER,PLASTIC & 01 & 19 & 9,375 & & & 317 & 2,932 & & \\
\hline PRIMARY METALS & 02 & 8 & 4,813 & & & 96 & 471 & & \\
\hline PROCESSED FOODS & 03 & 94 & 33,865 & & & 1,059 & 10,081 & & \\
\hline PAPER PRODUCTS & 04 & 9 & 7,653 & & & 848 & 4,264 & & \\
\hline PETROLEUM PRODUCTS & 05 & 14 & 4,714 & & & 539 & 1,350 & 363 & \\
\hline STONE,CLAY,GLASS,CONCRETE & 06 & 64 & 21,480 & & & 2,275 & 4,826 & & \\
\hline METAL DURABLE & 07 & 327 & 17,775 & & & 2,986 & 7,411 & & \\
\hline OTHER MANUFACTURING & 08 & 531 & 27,162 & & & 4,350 & 9,962 & & \\
\hline AGRICULTURE & 09 & 106 & 17,394 & & & 1,438 & 3,798 & & \\
\hline MINING & 10 & 3 & 2,449 & & & 38 & 102 & & \\
\hline UTILITY & 11 & & 569 & & & 1,850 & 4,713 & & \\
\hline GOVERNMENT & 12 & 520 & 4,851 & & & 6,118 & 4,443 & & \\
\hline
\end{tabular}

FLEET INDICATOR=NON-FLEET, SIZE=HEAVY

FLEET INDICATOR=NON-FLEET, SIZE=MEDIUM

\begin{tabular}{|c|c|c|c|c|c|c|c|c|c|}
\hline SECTOR & SECTOR \# & GASOLINE & DIESEL & $\mathrm{LPG}$ & $\mathrm{CNG}$ & GASOLINE & DIESEL & $\mathrm{LPG}$ & $\mathrm{CNG}$ \\
\hline CHEMICALS,RUBBER,PLASTIC & 01 & 116 & 1,395 & & & 425 & 2,430 & & \\
\hline PRIMARY METALS & 02 & 33 & 1,079 & & & 90 & 451 & & \\
\hline PROCESSED FOODS & 03 & 596 & 4,725 & & & 1,772 & 1,597 & & \\
\hline PAPER PRODUCTS & 04 & 36 & 1,458 & & & 456 & 1,015 & & \\
\hline PETROLEUM PRODUCTS & 05 & 57 & 1,536 & & & 162 & 689 & 225 & \\
\hline STONE,CLAY,GLASS,CONCRETE & 06 & 451 & 7,977 & & & 3,459 & 4,424 & & \\
\hline METAL DURABLE & 07 & 925 & 4,944 & & & 5,240 & 9,201 & & \\
\hline OTHER MANUFACTURING & 08 & 378 & 4,588 & & & 5,631 & 3,996 & & \\
\hline AGRICULTURE & 09 & 333 & 8,142 & & & 2,442 & 2,062 & & \\
\hline MINING & 10 & 13 & 900 & & & 35 & 218 & & \\
\hline UTILITY & 11 & & 172 & & & 554 & 891 & & \\
\hline GOVERNMENT & 12 & & & & & & & & \\
\hline
\end{tabular}


Because we got so little alternative fuel data in the original breakouts, here are some other data that might be of help:

NUMBER OF TRUCKS BY SIZE, SECTOR AND FUEL TYPE

SIZE=MEDIUM SIZE=HEAVY

\begin{tabular}{|c|c|c|c|c|c|c|c|c|c|}
\hline SECTOR & SECTOR \# & GASOLINE & DIESEL & LPG & CNG & GASOLINE & DIESEL & LPG & CNG \\
\hline CHEMICALS,RUBBER,PLASTIC & 01 & 742 & 5,362 & 4 & 1 & 136 & 10,771 & & \\
\hline PRIMARY METALS & 02 & 186 & 922 & & & 41 & 5,892 & & \\
\hline PROCESSED FOODS & 03 & 2,831 & 11,678 & 208 & & 690 & 38,590 & & \\
\hline PAPER PRODUCTS & 04 & 1,304 & 5,279 & & 328 & 45 & 9,110 & & \\
\hline PETROLEUM PRODUCTS & 05 & 702 & 2,039 & 588 & & 71 & 6,250 & & \\
\hline STONE,CLAY,GLASS,CONCRETE & 06 & 5,735 & 9,250 & 3 & & 515 & 29,457 & & \\
\hline METAL DURABLE & 07 & 8,227 & 16,611 & 3 & & 1,252 & 22,719 & & \\
\hline OTHER MANUFACTURING & 08 & 9,981 & 13,958 & & & 909 & 31,750 & & \\
\hline AGRICULTURE & 09 & 3,880 & 5,860 & & & 440 & 25,536 & & \\
\hline MINING & 10 & 73 & 321 & & & 16 & 3,350 & & \\
\hline UTILITY & 11 & 2,404 & 5,603 & & & & 742 & & \\
\hline GOVERNMENT & 12 & 6,118 & 4,443 & 167 & & 520 & 4,851 & & \\
\hline
\end{tabular}

NUMBER OF TRUCKS BY SIZE, FLEET INDICATOR AND FUEL TYPE

SIZE=MEDIUM

SIZE=HEAVY

\begin{tabular}{lrrrrrrrr}
\hline & GASOLINE & \multicolumn{1}{c}{ DIESEL } & LPG & CNG & GASOLINE & DIESEL & LPG & \multicolumn{2}{c}{ CNG } \\
\hline FLEET & 21,914 & 54,352 & 680 & 413 & 1,731 & 152,101 & 5 & 4 \\
NON-FLEET & 20,268 & 26,973 & 295 & 1 & 2,940 & 36,917 & 0 & 48 \\
\hline TOTAL & 42,182 & 81,325 & 976 & 414 & 4,671 & 189,018 & 5 & 52 \\
\hline
\end{tabular}


FLEET INDICATOR=FLEET, SIZE=HEAVY, ENGTYP=GASOLINE

\begin{tabular}{|c|c|c|c|c|c|c|c|c|c|c|c|c|}
\hline & SECTOR \# & & & & & & & & & & & \\
\hline AGE & 1 & 2 & 3 & 4 & 5 & 6 & 7 & 8 & 9 & 10 & 11 & 12 \\
\hline NEW & 19 & & 94 & & & & 327 & 531 & 106 & & & 520 \\
\hline 1 YR OLD & & & 59 & & & 106 & 535 & & & & 199 & 513 \\
\hline 2 YR OLD & 111 & 14 & 361 & 57 & 24 & 128 & 673 & 234 & 131 & 5 & & 597 \\
\hline 3 YR OLD & 392 & & 130 & 31 & 18 & 68 & 315 & 71 & 128 & & & 454 \\
\hline 4 YR OLD & & & 368 & & & & & & & & & 377 \\
\hline 5 YR OLD & & & & & & & & & & & & 276 \\
\hline 6 YR OLD & & & & & & & & & & & 41 & 254 \\
\hline 7 YR OLD & & & & & & & 27 & 55 & & & & 316 \\
\hline 8 YR OLD & & & 75 & & & 252 & 358 & 171 & & & & 335 \\
\hline 9 YR OLD & & & & & & & & & & & & 325 \\
\hline $10+Y R$ OLD & 1,610 & 186 & 1,243 & 311 & 1,255 & 4,326 & 3,655 & 1,416 & 6,869 & 188 & 808 & 2,711 \\
\hline
\end{tabular}

FLEET INDICATOR=FLEET, SIZE = HEAVY, ENGTYP=DIESEL

\begin{tabular}{|c|c|c|c|c|c|c|c|c|c|c|c|c|}
\hline \multicolumn{13}{|c|}{ SECTOR \# } \\
\hline AGE & 1 & 2 & 3 & 4 & 5 & 6 & 7 & 8 & 9 & 10 & 11 & 12 \\
\hline NEW & 9,375 & 4,813 & 33,865 & 7,653 & 4,714 & 21,480 & 17,775 & 27,162 & 17,394 & 2,449 & 569 & 4,851 \\
\hline 1 YR OLD & 8,782 & 5,173 & 29,462 & 8,223 & 4,530 & 21,184 & 19,499 & 27,402 & 17,479 & 1,916 & 1,110 & 4,782 \\
\hline 2 YR OLD & 10,255 & 5,419 & 32,070 & 10,602 & 6,370 & 25,370 & 20,637 & 27,037 & 21,945 & 1,689 & 1,647 & 5,567 \\
\hline 3 YR OLD & 8,226 & 4,790 & 23,089 & 6,182 & 4,817 & 19,133 & 17,447 & 19,962 & 14,379 & 1,419 & 911 & 4,234 \\
\hline 4 YR OLD & 7,889 & 3,908 & 18,889 & 5,035 & 3,479 & 14,160 & 11,837 & 14,341 & 12,330 & 1,060 & 972 & 3,518 \\
\hline 5 YR OLD & 4,414 & 1,722 & 14,956 & 2,330 & 2,345 & 8,164 & 8,992 & 8,221 & 8,644 & 885 & 852 & 2,578 \\
\hline 6 YR OLD & 4,430 & 1,800 & 13,000 & 2,304 & 3,733 & 9,683 & 7,233 & 7,226 & 8,011 & 1,059 & 908 & 2,366 \\
\hline 7 YR OLD & 4,442 & 1,875 & 11,086 & 2,197 & 2,946 & 12,592 & 10,153 & 9,978 & 8,029 & 922 & 722 & 2,945 \\
\hline 8 YR OLD & 2,972 & 3,252 & 8,318 & 2,216 & 3,132 & 15,053 & 9,806 & 8,000 & 9,296 & 642 & 1,011 & 3,126 \\
\hline 9 YR OLD & 3,681 & 1,994 & 7,952 & 1,800 & 2,833 & 15,629 & 8,360 & 8,318 & 8,716 & 797 & 1,496 & 3,026 \\
\hline $10+$ YR OLD & 18,143 & 8,042 & 27,535 & 7,106 & 18,022 & 112,051 & 59,993 & 31,588 & 56,954 & 7,693 & 6,576 & 25,275 \\
\hline
\end{tabular}


NUMBER OF TRUCKS BY FLEET INDICATOR, SIZE, FUEL TYPE, SECTOR, AND AGE (CONTINUTED)

FLEET INDICATOR=FLEET, SIZE $=$ HEAVY, ENGTYP=LPG

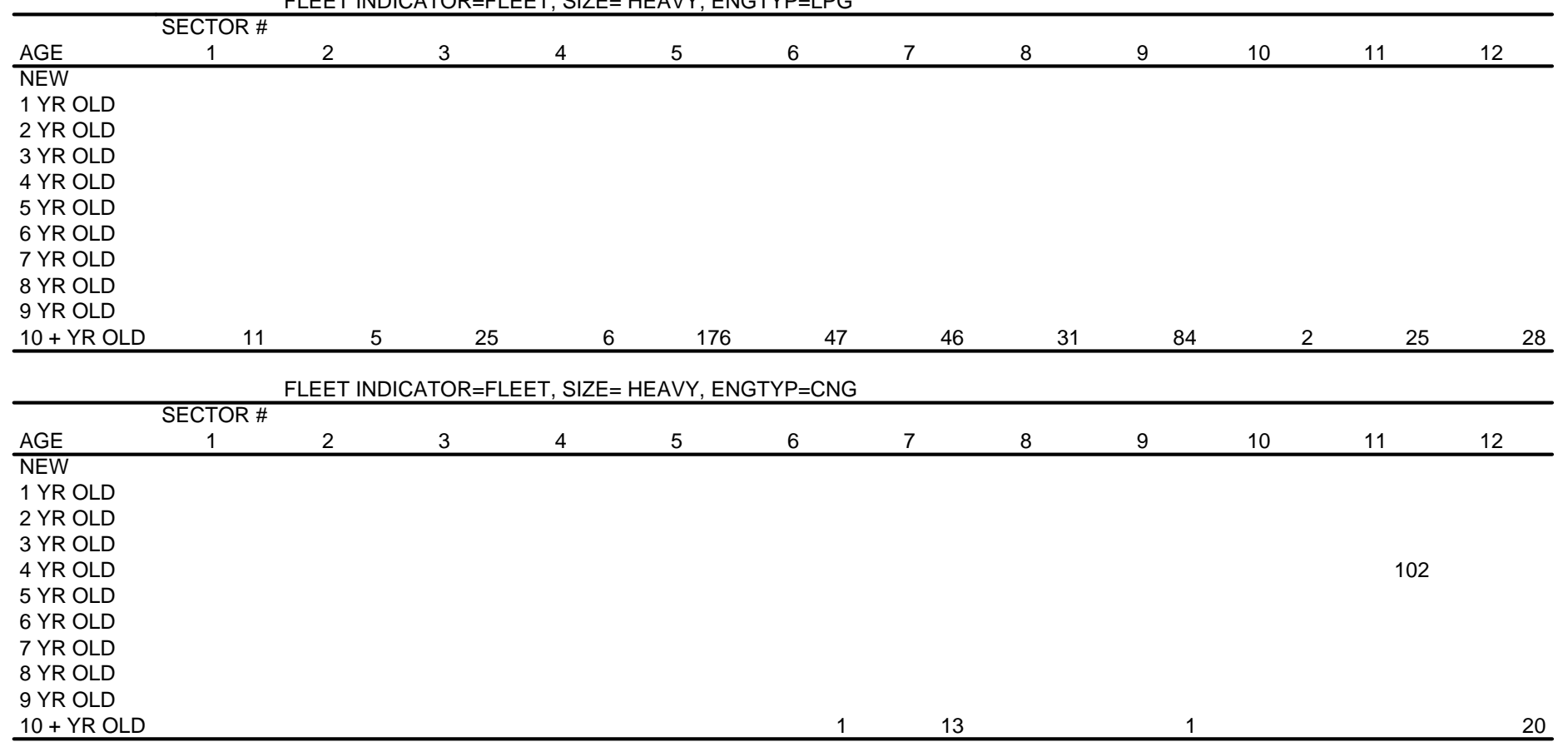


FLEET INDICATOR=FLEET, SIZE=MEDIUM, ENGTYP=GASOLINE

\begin{tabular}{|c|c|c|c|c|c|c|c|c|c|c|c|c|}
\hline \multicolumn{13}{|c|}{ SECTOR \# } \\
\hline AGE & 1 & 2 & 3 & 4 & 5 & 6 & 7 & 8 & 9 & 10 & 11 & 12 \\
\hline NEW & 317 & 96 & 1,059 & 848 & 539 & 2,275 & 2,986 & 4,350 & 1,438 & 38 & 1,850 & 6,118 \\
\hline 1 YR OLD & 155 & 300 & 3,003 & 505 & 127 & 2,599 & 3,588 & 1,717 & 1,391 & 29 & 3,094 & 6,637 \\
\hline 2 YR OLD & 2,042 & 267 & 1,379 & 432 & 503 & 3,392 & 4,688 & 6,939 & 1,104 & 39 & 3,309 & 9,948 \\
\hline 3 YR OLD & 412 & 158 & 2,592 & 524 & 618 & 1,828 & 4,278 & 4,947 & 1,811 & 62 & 2,503 & 7,068 \\
\hline 4 YR OLD & 932 & 207 & 937 & 146 & 530 & 2,285 & 3,291 & 3,807 & 819 & 19 & 2,540 & 5,583 \\
\hline 5 YR OLD & 586 & 72 & 1,430 & 76 & 126 & 949 & 4,847 & 2,880 & 739 & 29 & 2,790 & 4,499 \\
\hline 6 YR OLD & 634 & 73 & 1,468 & 472 & 1,016 & 3,519 & 2,740 & 2,756 & 1,645 & 29 & 2,880 & 5,717 \\
\hline 7 YR OLD & 1,019 & 539 & 1,735 & 572 & 907 & 2,607 & 7,987 & 6,013 & 1,273 & 37 & 3,164 & 7,320 \\
\hline 8 YR OLD & 2,893 & 84 & 1,527 & 174 & 351 & 3,813 & 5,542 & 6,354 & 2,796 & 22 & 6,087 & 7,795 \\
\hline 9 YR OLD & 1,094 & 694 & 2,238 & 513 & 833 & 2,061 & 3,258 & 6,257 & 3,009 & 59 & 6,091 & 7,573 \\
\hline $10+$ YR OLD & 13,042 & 3,398 & 23,235 & 3,641 & 11,723 & 44,501 & 46,729 & 30,250 & 48,317 & 1,437 & 18,645 & 80,226 \\
\hline \multicolumn{13}{|c|}{ FLEET INDICATOR=FLEET, SIZE=MEDIUM, ENGTYP=DIESEL } \\
\hline & CTOR \# & & & & & & & & & & & \\
\hline AGE & 1 & 2 & 3 & 4 & 5 & 6 & 7 & 8 & 9 & 10 & 11 & 12 \\
\hline NEW & 2,932 & 471 & 10,081 & 4,264 & 1,350 & 4,826 & 7,411 & 9,962 & 3,798 & 102 & 4,713 & 4,443 \\
\hline 1 YR OLD & 2,344 & 913 & 7,595 & 2,434 & 1,679 & 2,842 & 13,859 & 8,886 & 4,610 & 191 & 5,203 & 4,820 \\
\hline 2 YR OLD & 4,934 & 1,031 & 17,658 & 2,287 & 2,251 & 7,937 & 18,226 & 20,522 & 6,303 & 201 & 10,125 & 7,225 \\
\hline 3 YR OLD & 2,991 & 578 & 9,428 & 1,304 & 2,088 & 6,182 & 9,060 & 11,718 & 5,397 & 128 & 3,492 & 5,133 \\
\hline 4 YR OLD & 1,618 & 837 & 6,800 & 1,474 & 1,528 & 3,436 & 8,158 & 11,581 & 3,025 & 165 & 3,685 & 4,055 \\
\hline 5 YR OLD & 2,255 & 230 & 6,487 & 1,497 & 725 & 927 & 3,745 & 6,040 & 3,448 & 102 & 4,895 & 3,267 \\
\hline 6 YR OLD & 2,220 & 145 & 7,209 & 722 & 1,335 & 3,027 & 5,105 & 6,298 & 4,267 & 57 & 8,230 & 4,152 \\
\hline 7 YR OLD & 3,619 & 2,015 & 5,872 & 380 & 2,218 & 6,800 & 9,730 & 14,783 & 3,701 & 106 & 5,614 & 5,316 \\
\hline 8 YR OLD & 3,510 & 1,191 & 5,084 & 380 & 1,053 & 3,327 & 5,327 & 15,174 & 2,643 & 56 & 5,017 & 5,661 \\
\hline 9 YR OLD & 3,730 & 87 & 11,175 & 477 & 1,661 & 2,703 & 4,316 & 7,406 & 3,332 & 246 & 2,771 & 5,500 \\
\hline $10+$ YR OLD & 8,149 & 2,439 & 30,893 & 2,095 & 10,128 & 20,490 & 28,401 & 16,827 & 22,635 & 398 & 16,653 & 58,263 \\
\hline
\end{tabular}


NUMBER OF TRUCKS BY FLEET INDICATOR, SIZE, FUEL TYPE, SECTOR, AND AGE (CONTINUTED)

FLEET INDICATOR=FLEET, SIZE=MEDIUM, ENGTYP=LPG

\begin{tabular}{|c|c|c|c|c|c|c|c|c|c|c|c|c|}
\hline 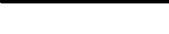 & SECTOR \# & & & & & & & & & & & \\
\hline AGE & 1 & 2 & 3 & 4 & 5 & 6 & 7 & 8 & 9 & 10 & 11 & 12 \\
\hline NEW & & & & & 363 & & & & & & & 167 \\
\hline 1 YR OLD & & & & & & & & & & & & 181 \\
\hline 2 YR OLD & 474 & & 134 & & 461 & 3 & 5 & & & & & 271 \\
\hline 3 YR OLD & 10 & 4 & 60 & 4 & 744 & 29 & 81 & 21 & 34 & 1 & & 193 \\
\hline 4 YR OLD & 1 & & 929 & & 1,006 & 4 & 3 & 9 & 2 & & & 152 \\
\hline 5 YR OLD & 3 & & 366 & & 695 & 6 & 7 & 5 & 4 & & & 123 \\
\hline 6 YR OLD & & & & & 1,506 & 86 & 42 & 29 & 48 & & & 156 \\
\hline 7 YR OLD & & & & & & & & & & & & 199 \\
\hline 8 YR OLD & & & & & 818 & & & & 330 & & & 212 \\
\hline 9 YR OLD & & & & & 809 & & 7 & & & & & 206 \\
\hline \multirow[t]{3}{*}{$10+$ YR OLD } & 1,322 & 279 & 537 & 253 & 1,450 & 549 & 2,913 & 1,684 & 1,906 & 25 & 558 & 2,185 \\
\hline & & EET IND & $\mathrm{TOR}=\mathrm{FL}$ & $T$, SIZE $=1$ & DIUM, El & $\mathrm{YYP}=\mathrm{CNC}$ & & & & & & \\
\hline & SECTOR \# & & & & & & & & & & & \\
\hline AGE & 1 & 2 & 3 & 4 & 5 & 6 & 7 & 8 & 9 & 10 & 11 & 12 \\
\hline 1 YR OLD & & & & & & & & & & & & 24 \\
\hline 2 YR OLD & & & 1 & & & & 1 & & & & & 37 \\
\hline 3 YR OLD & 1 & & 1 & & & 1 & 1 & 1 & 1 & & & 26 \\
\hline 4 YR OLD & & & & & & 1 & & 1 & & & & 21 \\
\hline 5 YR OLD & & & & & & 1 & 1 & 1 & 1 & & & 17 \\
\hline 6 YR OLD & & & & & & & & & & & & 21 \\
\hline 7 YR OLD & & & & & & & & & & & & 27 \\
\hline 8 YR OLD & & & & & & & & & & & & 29 \\
\hline 9 YR OLD & & & & & & & 1 & & & & & 28 \\
\hline $10+Y R$ OLD & 21 & 10 & 47 & 10 & 18 & 81 & 219 & 303 & 258 & 417 & & 296 \\
\hline
\end{tabular}


NUMBER OF TRUCKS BY FLEET INDICATOR, SIZE, FUEL TYPE, SECTOR, AND AGE (CONTINUTED)

5

FLEET INDICATOR=NON-FLEET, SIZE $=$ HEAVY, ENGTYP=GASOLINE

\begin{tabular}{|c|c|c|c|c|c|c|c|c|c|c|c|c|}
\hline \multicolumn{13}{|c|}{ SECTOR \# } \\
\hline AGE & 1 & 2 & 3 & 4 & 5 & 6 & 7 & 8 & 9 & 10 & 11 & 12 \\
\hline NEW & 116 & 33 & 596 & 36 & 57 & 451 & 925 & 378 & 333 & 13 & & \\
\hline 1 YR OLD & 128 & 169 & 366 & 141 & 53 & 377 & 615 & 571 & 376 & 12 & & \\
\hline 2 YR OLD & 327 & 41 & 543 & 153 & 71 & 442 & 1,444 & 785 & 387 & 16 & & \\
\hline 3 YR OLD & 623 & 22 & 503 & 120 & 98 & 456 & 570 & 689 & 424 & 9 & & \\
\hline 4 YR OLD & 169 & 39 & 284 & 90 & 68 & 399 & 631 & 321 & 479 & 65 & & \\
\hline 5 YR OLD & 61 & 105 & 193 & 30 & 50 & 292 & 340 & 657 & 458 & 11 & & \\
\hline 6 YR OLD & 71 & 33 & 402 & 35 & 58 & 435 & 497 & 340 & 321 & 30 & & \\
\hline 7 YR OLD & 61 & 29 & 309 & 30 & 50 & 250 & 461 & 189 & 315 & 11 & & \\
\hline 8 YR OLD & 110 & 38 & 439 & 39 & 107 & 306 & 522 & 322 & 358 & 16 & & \\
\hline 9 YR OLD & 73 & 34 & 202 & 36 & 273 & 453 & 625 & 253 & 737 & 13 & & \\
\hline $10+$ YR OLD & 7,080 & 2,787 & 12,504 & 2,752 & 6,207 & 31,058 & 28,682 & 16,957 & 65,971 & 1,587 & 256 & \\
\hline \multicolumn{13}{|c|}{ FLEET INDICATOR=NON-FLEET, SIZE=HEAVY, ENGTYP=DIESEL } \\
\hline & CTOR \# & & & & & & & & & & & \\
\hline AGE & 1 & 2 & 3 & 4 & 5 & 6 & 7 & 8 & 9 & 10 & 11 & 12 \\
\hline NEW & 1,395 & 1,079 & 4,725 & 1,458 & 1,536 & 7,977 & 4,944 & 4,588 & 8,142 & 900 & 172 & \\
\hline 1 YR OLD & 1,726 & 1,198 & 4,936 & 1,690 & 1,871 & 7,335 & 5,511 & 3,694 & 8,240 & 410 & & \\
\hline 2 YR OLD & 2,806 & 1,649 & 6,424 & 1,982 & 1,884 & 7,662 & 8,436 & 6,695 & 8,585 & 572 & & \\
\hline 3 YR OLD & 1,971 & 1,394 & 4,379 & 929 & 1,257 & 6,881 & 7,064 & 6,502 & 8,189 & 700 & 117 & \\
\hline 4 YR OLD & 2,604 & 1,648 & 4,256 & 1,602 & 1,139 & 5,625 & 7,170 & 6,702 & 7,652 & 787 & & \\
\hline 5 YR OLD & 2,405 & 992 & 4,842 & 1,773 & 1,225 & 4,809 & 5,938 & 5,759 & 7,285 & 499 & 102 & \\
\hline 6 YR OLD & 1,612 & 1,249 & 3,530 & 1,201 & 887 & 4,446 & 4,578 & 4,824 & 6,854 & 475 & 245 & \\
\hline 7 YR OLD & 1,494 & 2,082 & 4,839 & 2,208 & 1,028 & 9,304 & 8,020 & 7,337 & 10,054 & 683 & 236 & \\
\hline 8 YR OLD & 2,482 & 2,394 & 4,530 & 1,823 & 1,982 & 11,274 & 9,227 & 6,249 & 13,697 & 798 & & \\
\hline 9 YR OLD & 2,170 & 1,310 & 5,287 & 1,191 & 1,733 & 11,504 & 8,396 & 6,711 & 13,465 & 850 & 190 & \\
\hline $10+$ YR OLD & 14,109 & 10,528 & 20,755 & 5,127 & 12,127 & 124,413 & 65,951 & 34,080 & 132,060 & 6,672 & 1,521 & \\
\hline
\end{tabular}


NUMBER OF TRUCKS BY FLEET INDICATOR, SIZE, FUEL TYPE, SECTOR, AND AGE (CONTINUTED)

6

FLEET INDICATOR=NON-FLEET, SIZE=HEAVY, ENGTYP=LPG

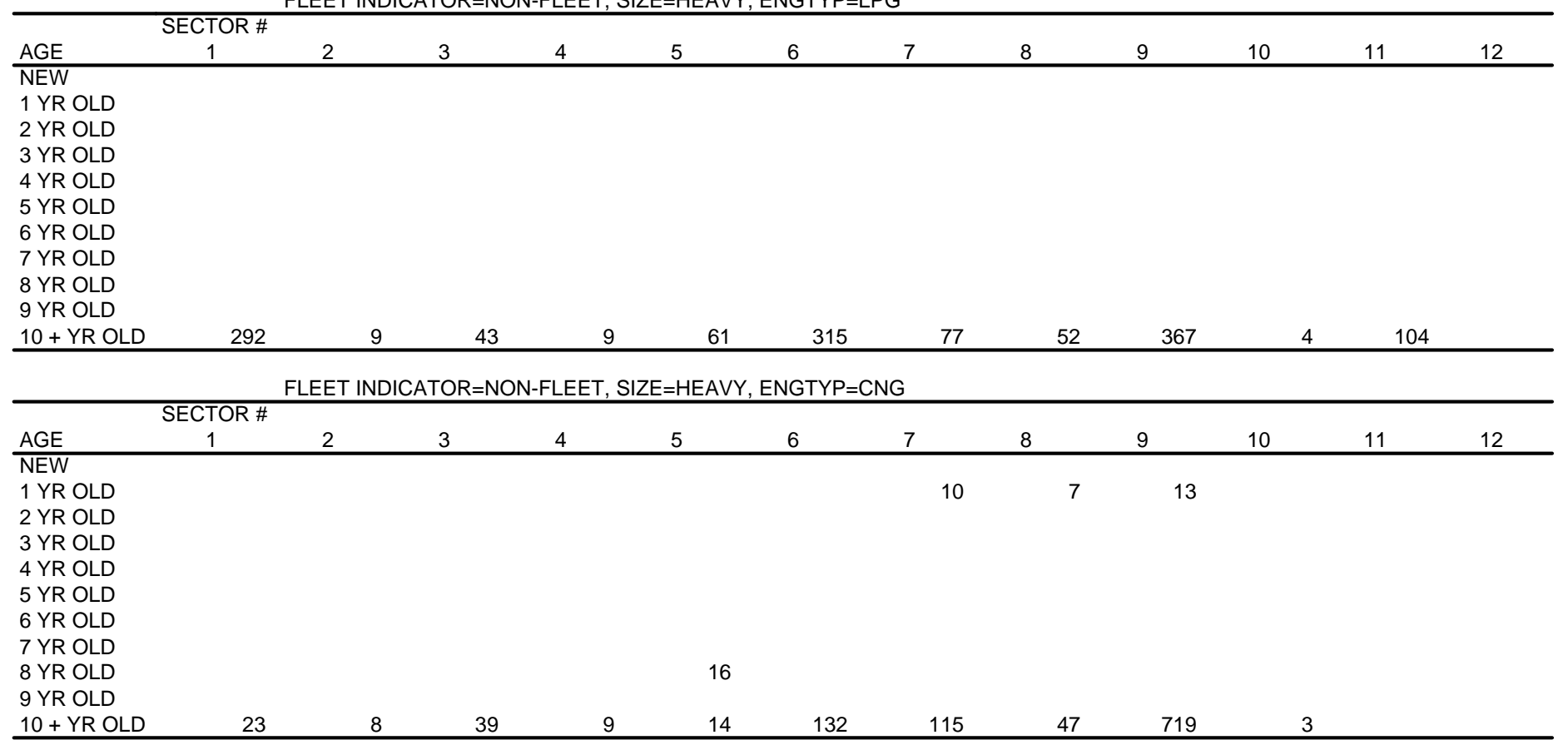


FLEET INDICATOR=NON-FLEET, SIZE=MEDIUM, ENGTYP=GASOLINE

\begin{tabular}{|c|c|c|c|c|c|c|c|c|c|c|c|c|}
\hline \multicolumn{13}{|c|}{ SECTOR \# } \\
\hline AGE & 1 & 2 & 3 & 4 & 5 & 6 & 7 & 8 & 9 & 10 & 11 & 12 \\
\hline NEW & 425 & 90 & 1,772 & 456 & 162 & 3,459 & 5,240 & 5,631 & 2,442 & 35 & 554 & \\
\hline 1 YR OLD & 1,069 & 197 & 947 & 515 & 272 & 4,193 & 5,012 & 3,569 & 2,445 & 62 & 1,038 & \\
\hline 2 YR OLD & 427 & 1,019 & 3,849 & 532 & 365 & 4,226 & 7,862 & 6,783 & 3,466 & 169 & 364 & \\
\hline 3 YR OLD & 496 & 380 & 1,365 & 242 & 527 & 4,118 & 8,776 & 6,350 & 3,229 & 35 & 319 & \\
\hline 4 YR OLD & 467 & 575 & 1,316 & 463 & 267 & 1,709 & 6,929 & 5,765 & 1,147 & 33 & 435 & \\
\hline 5 YR OLD & 680 & 687 & 1,388 & 58 & 543 & 2,415 & 3,035 & 2,878 & 2,431 & 134 & 267 & \\
\hline 6 YR OLD & 1,063 & 376 & 786 & 281 & 1,302 & 2,084 & 6,323 & 1,678 & 4,941 & 46 & 1,437 & \\
\hline 7 YR OLD & 597 & 1,717 & 2,113 & 160 & 534 & 3,489 & 6,951 & 5,073 & 2,395 & 145 & 714 & \\
\hline 8 YR OLD & 1,209 & 837 & 4,063 & 729 & 710 & 5,991 & 12,509 & 7,641 & 5,680 & 258 & 1,193 & \\
\hline 9 YR OLD & 2,686 & 843 & 4,636 & 420 & 2,388 & 7,766 & 11,282 & 5,777 & 7,501 & 82 & 514 & \\
\hline $10+Y R$ OLD & 26,955 & 13,299 & 38,155 & 6,005 & 24,148 & 152,393 & 142,779 & 68,048 & 384,861 & 4,462 & 7,059 & \\
\hline \multicolumn{13}{|c|}{ FLEET INDICATOR=NON-FLEET, SIZE=MEDIUM, ENGTYP=DIESEL } \\
\hline & CTOR \# & & & & & & & & & & & \\
\hline AGE & 1 & 2 & 3 & 4 & 5 & 6 & 7 & 8 & 9 & 10 & 11 & 12 \\
\hline NEW & 2,430 & 451 & 1,597 & 1,015 & 689 & 4,424 & 9,201 & 3,996 & 2,062 & 218 & 891 & \\
\hline 1 YR OLD & 2,157 & 1,233 & 2,939 & 1,176 & 1,561 & 4,035 & 13,061 & 4,566 & 4,365 & 65 & 507 & \\
\hline 2 YR OLD & 1,373 & 441 & 3,174 & 1,204 & 1,423 & 5,081 & 13,519 & 5,779 & 5,103 & 74 & 966 & \\
\hline 3 YR OLD & 1,063 & 526 & 3,020 & 418 & 1,327 & 5,194 & 9,125 & 6,705 & 3,761 & 243 & 166 & \\
\hline 4 YR OLD & 1,288 & 264 & 3,605 & 269 & 1,764 & 4,295 & 7,430 & 1,918 & 3,637 & 48 & 486 & \\
\hline 5 YR OLD & 770 & 1,212 & 3,435 & 579 & 1,287 & 1,712 & 5,239 & 3,206 & 4,357 & 34 & 167 & \\
\hline 6 YR OLD & 2,000 & 199 & 2,566 & 349 & 1,291 & 2,749 & 6,310 & 3,709 & 6,152 & 145 & 615 & \\
\hline 7 YR OLD & 1,745 & 501 & 2,389 & 847 & 1,590 & 5,378 & 6,801 & 5,749 & 4,322 & 36 & 468 & \\
\hline 8 YR OLD & 1,676 & 493 & 1,882 & 1,588 & 1,863 & 5,665 & 7,518 & 3,110 & 4,036 & 52 & 1,507 & \\
\hline 9 YR OLD & 1,908 & 897 & 3,763 & 394 & 1,258 & 4,993 & 8,344 & 2,219 & 6,730 & 108 & 963 & \\
\hline $10+$ YR OLD & 7,430 & 3,885 & 12,679 & 3,557 & 8,355 & 41,880 & 41,760 & 23,821 & 50,433 & 1,063 & 2,690 & \\
\hline
\end{tabular}


NUMBER OF TRUCKS BY FLEET INDICATOR, SIZE, FUEL TYPE, SECTOR, AND AGE (CONTINUTED)

8

FLEET INDICATOR=NON-FLEET, SIZE=MEDIUM, ENGTYP=LPG

\begin{tabular}{|c|c|c|c|c|c|c|c|c|c|c|c|c|}
\hline & CTOR \# & & & & & & & & & & & \\
\hline AGE & 1 & 2 & 3 & 4 & 5 & 6 & 7 & 8 & 9 & 10 & 11 & 12 \\
\hline NEW & & & & & 225 & & & & & & & \\
\hline \multicolumn{13}{|l|}{1 YR OLD } \\
\hline 2 YR OLD & & & 260 & 1 & 1,156 & 3 & 7 & & & & & \\
\hline 3 YR OLD & & & & & 713 & & & & & & & \\
\hline 4 YR OLD & & & 2 & & 21 & 4 & & 2 & 1 & & & \\
\hline 5 YR OLD & 175 & & & & 244 & 3 & 5 & 3 & & & & \\
\hline 6 YR OLD & & & & & 904 & & & & & & & \\
\hline \multicolumn{13}{|l|}{7 YR OLD } \\
\hline 8 YR OLD & & & & & 491 & & & & & & & \\
\hline 9 YR OLD & 188 & & 13 & & 274 & 5 & 9 & 4 & 102 & & & \\
\hline \multirow[t]{2}{*}{$10+$ YR OLD } & 1,124 & 217 & 500 & 40 & 2,749 & 666 & 851 & 326 & 3,576 & 110 & 333 & \\
\hline \multirow{2}{*}{\multicolumn{13}{|c|}{$\begin{array}{lc}\text { FLEET INDICATOR=NON-FLEET, SIZE=MEDIUM, ENGTYP=CNG } \\
\text { SECTOR \# }\end{array}$}} \\
\hline & & & & & & & & & & & & \\
\hline AGE & 1 & 2 & 3 & 4 & 5 & 6 & 7 & 8 & 9 & 10 & 11 & 12 \\
\hline \multicolumn{13}{|l|}{$\begin{array}{l}\text { NEW } \\
1 \text { YR OLD }\end{array}$} \\
\hline 2 YR OLD & & & 2 & & & & 1 & & & & & \\
\hline 3 YR OLD & & & & & & & & & & & & \\
\hline 4 YR OLD & & & & & & 1 & & & & & & \\
\hline 5 YR OLD & & & & & & & 1 & & & & & \\
\hline 6 YR OLD & & & & & & & & & & & & \\
\hline 7 YR OLD & & & & & & & & & & & & \\
\hline 8 YR OLD & & & & & & & & & 22 & & & \\
\hline 9 YR OLD & & & 2 & & & 1 & 1 & 1 & 1 & & & \\
\hline $10+$ YR OLD & 121 & 28 & 132 & 29 & 49 & 313 & 376 & 306 & 1,051 & 11 & & \\
\hline
\end{tabular}


VIUS 1997

TRUCKS BY SECTOR, SIZE CLASS, AGE, AND FLEET INDICATOR

FLEET $=1$, HEAVY

\begin{tabular}{|c|c|c|c|c|c|c|c|c|c|c|c|c|}
\hline MDLYR / SECTOR & 1 & 2 & 3 & 4 & 5 & 6 & 7 & 8 & 9 & 10 & 11 & 12 \\
\hline NEW & 9,395 & 4,821 & 33,959 & 7,661 & 4,729 & 21,543 & 18,102 & 27,693 & 17,500 & 2,453 & 606 & 5,380 \\
\hline 1 YR OLD & 8,923 & 5,218 & 29,603 & 8,224 & 4,636 & 21,289 & 20,035 & 27,507 & 17,500 & 1,917 & 1,411 & 5,304 \\
\hline 2 YR OLD & 10,366 & 5,433 & 32,513 & 10,659 & 6,419 & 25,548 & 21,555 & 27,270 & 22,075 & 1,694 & 1,697 & 6,174 \\
\hline 3 YR OLD & 8,617 & 4,795 & 23,219 & 6,213 & 4,835 & 19,201 & 17,763 & 20,069 & 14,507 & 1,421 & 911 & 4,696 \\
\hline 4 YR OLD & 7,931 & 3,975 & 19,257 & 5,035 & 3,480 & 14,200 & 11,962 & 14,419 & 12,341 & 1,060 & 1,074 & 3,902 \\
\hline 5 YR OLD & 4,513 & 1,758 & 14,993 & 2,330 & 2,345 & 8,347 & 9,060 & 8,304 & 8,670 & 885 & 1,010 & 2,859 \\
\hline 6 YR OLD & 4,462 & 1,801 & 13,004 & 2,361 & 3,735 & 9,772 & 7,405 & 7,232 & 8,069 & 1,078 & 949 & 2,624 \\
\hline 7 YR OLD & 4,449 & 1,878 & 11,119 & 2,200 & 2,951 & 12,615 & 10,179 & 10,033 & 8,094 & 923 & 863 & 3,267 \\
\hline 8 YR OLD & 3,060 & 3,255 & 8,394 & 2,219 & 3,137 & 15,306 & 10,165 & 8,172 & 9,328 & 644 & 1,011 & 3,467 \\
\hline 9 YR OLD & 3,721 & 1,994 & 8,025 & 1,800 & 2,833 & 15,662 & 8,487 & 8,335 & 8,717 & 797 & 1,617 & 3,357 \\
\hline $10+$ YR OLD & 19,765 & 8,234 & 28,803 & 7,423 & 19,453 & 116,425 & 63,707 & 33,035 & 63,908 & 7,883 & 7,409 & 28,033 \\
\hline \multicolumn{13}{|l|}{ FLEET=1, MEDIUM } \\
\hline MDLYR / SECTOR & 1 & 2 & 3 & 4 & 5 & 6 & 7 & 8 & 9 & 10 & 11 & 12 \\
\hline NEW & 3,251 & 566 & 11,286 & 5,440 & 2,252 & 7,102 & 10,399 & 14,312 & 5,236 & 140 & 6,624 & 10,750 \\
\hline 1 YR OLD & 2,500 & 1,213 & 10,765 & 2,939 & 2,096 & 5,444 & 17,451 & 10,606 & 6,006 & 220 & 8,305 & 11,663 \\
\hline 2 YR OLD & 7,450 & 1,298 & 19,172 & 2,719 & 3,215 & 11,333 & 22,920 & 27,470 & 7,410 & 241 & 13,434 & 17,481 \\
\hline 3 YR OLD & 3,413 & 740 & 12,081 & 1,833 & 3,451 & 8,040 & 13,420 & 16,687 & 7,243 & 192 & 6,145 & 12,419 \\
\hline 4 YR OLD & 2,550 & 1,045 & 8,666 & 1,621 & 3,064 & 5,726 & 11,453 & 15,397 & 3,847 & 184 & 6,231 & 9,810 \\
\hline 5 YR OLD & 2,844 & 303 & 8,283 & 1,574 & 1,546 & 1,883 & 8,600 & 8,925 & 4,191 & 130 & 8,525 & 7,906 \\
\hline 6 YR OLD & 2,864 & 223 & 8,700 & 1,199 & 3,857 & 6,633 & 7,887 & 9,083 & 5,960 & 88 & 12,226 & 10,045 \\
\hline 7 YR OLD & 4,642 & 2,554 & 7,936 & 951 & 3,617 & 9,413 & 17,719 & 20,796 & 4,974 & 144 & 8,784 & 12,863 \\
\hline 8 YR OLD & 6,404 & 1,276 & 6,789 & 554 & 2,221 & 7,141 & 10,993 & 21,529 & 5,769 & 77 & 11,106 & 13,698 \\
\hline 9 YR OLD & 4,826 & 782 & 13,422 & 991 & 3,303 & 4,768 & 7,581 & 13,667 & 6,346 & 306 & 8,998 & 13,307 \\
\hline $10+$ YR OLD & 22,534 & 6,126 & 54,712 & 5,999 & 23,319 & 65,621 & 78,263 & 49,065 & 73,116 & 2,277 & 35,857 & 140,971 \\
\hline
\end{tabular}


FLEET $=2$ (NON-FLEET), HEAVY

\begin{tabular}{|c|c|c|c|c|c|c|c|c|c|c|c|c|}
\hline MDLYR / SECTOR & 1 & 2 & 3 & 4 & 5 & 6 & 7 & 8 & 9 & 10 & 11 & 12 \\
\hline NEW & 1,512 & 1,112 & 5,322 & 1,494 & 1,593 & 8,429 & 5,870 & 4,967 & 8,521 & 913 & 172 & \\
\hline 1 YR OLD & 1,856 & 1,368 & 5,308 & 1,832 & 1,927 & 7,722 & 6,136 & 4,271 & 8,629 & 423 & & \\
\hline 2 YR OLD & 3,133 & 1,691 & 6,967 & 2,135 & 1,955 & 8,103 & 9,881 & 7,480 & 8,972 & 588 & 139 & \\
\hline 3 YR OLD & 2,594 & 1,416 & 4,881 & 1,078 & 1,356 & 7,337 & 7,634 & 7,192 & 8,613 & 708 & 117 & \\
\hline 4 YR OLD & 2,773 & 1,688 & 4,541 & 1,693 & 1,208 & 6,023 & 7,801 & 7,024 & 8,131 & 852 & & \\
\hline 5 YR OLD & 2,465 & 1,098 & 5,035 & 1,803 & 1,274 & 5,101 & 6,279 & 6,417 & 7,743 & 511 & 102 & \\
\hline 6 YR OLD & 1,704 & 1,283 & 3,932 & 1,236 & 945 & 4,881 & 5,076 & 5,164 & 7,176 & 506 & 245 & \\
\hline 7 YR OLD & 1,556 & 2,111 & 5,148 & 2,239 & 1,078 & 9,595 & 8,482 & 7,526 & 10,370 & 695 & 272 & \\
\hline 8 YR OLD & 2,592 & 2,432 & 4,969 & 1,863 & 2,104 & 11,580 & 9,750 & 6,572 & 14,055 & 813 & & \\
\hline 9 YR OLD & 2,243 & 1,345 & 5,489 & 1,227 & 2,006 & 11,957 & 9,021 & 6,964 & 14,202 & 864 & 207 & \\
\hline $10+$ YR OLD & 21,503 & 13,332 & 33,341 & 7,897 & 18,409 & 155,918 & 94,825 & 51,136 & 199,117 & 8,266 & 1,881 & \\
\hline \multicolumn{13}{|c|}{ FLEET=2(NON-FLEET), MEDIUM } \\
\hline MDLYR / SECTOR & 1 & 2 & 3 & 4 & 5 & 6 & 7 & 8 & 9 & 10 & 11 & 12 \\
\hline NEW & 2,858 & 541 & 3,431 & 1,471 & 1,076 & 7,886 & 14,444 & 9,627 & 4,505 & 254 & 1,445 & \\
\hline 1 YR OLD & 3,429 & 1,431 & 3,928 & 1,693 & 2,325 & 8,239 & 18,086 & 8,143 & 6,824 & 127 & 1,815 & \\
\hline 2 YR OLD & 1,923 & 1,460 & 7,285 & 1,736 & 2,944 & 9,310 & 21,388 & 12,566 & 8,571 & 243 & 1,332 & \\
\hline 3 YR OLD & 1,790 & 906 & 4,557 & 659 & 2,566 & 9,315 & 17,907 & 13,055 & 6,990 & 278 & 581 & \\
\hline 4 YR OLD & 1,754 & 839 & 4,923 & 731 & 2,053 & 6,008 & 14,360 & 7,685 & 4,784 & 82 & 1,063 & \\
\hline 5 YR OLD & 1,625 & 1,899 & 4,822 & 637 & 2,074 & 4,131 & 8,280 & 6,088 & 6,788 & 168 & 434 & \\
\hline 6 YR OLD & 3,069 & 578 & 3,592 & 633 & 3,498 & 5,023 & 12,659 & 5,406 & 11,125 & 192 & 2,052 & \\
\hline 7 YR OLD & 2,342 & 2,219 & 4,662 & 1,129 & 2,488 & 8,868 & 13,915 & 10,822 & 6,717 & 181 & 1,481 & \\
\hline 8 YR OLD & 3,031 & 1,332 & 6,004 & 2,319 & 3,480 & 11,676 & 20,143 & 10,767 & 9,741 & 311 & 2,702 & \\
\hline 9 YR OLD & 4,782 & 1,740 & 8,414 & 815 & 3,919 & 12,765 & 19,636 & 8,000 & 14,333 & 190 & 1,536 & \\
\hline $10+$ YR OLD & 35,630 & 17,429 & 51,467 & 9,630 & 35,302 & 195,251 & 185,766 & 92,500 & 439,921 & 5,646 & 10,082 & \\
\hline
\end{tabular}


VIUS 1997

AVERAGE ANNUAL VMT PER TRUCK BY SECTOR, SIZE, FUEL TYPE AND AGE

SIZE=HEAVY, ENGTYP=GASOLINE

\begin{tabular}{|c|c|c|c|c|c|c|c|c|c|c|c|c|}
\hline & CTOR \# & & & & & & & & & & & \\
\hline AGE & 1 & 2 & 3 & 4 & 5 & 6 & 7 & 8 & 9 & 10 & 11 & $12^{*}$ \\
\hline NEW & 13,906 & 17,178 & 21,371 & 16,636 & 17,178 & 16,639 & 14,117 & 18,757 & 15,668 & 17,178 & & 16,925 \\
\hline 1 YR OLD & 16,619 & 14,249 & 24,148 & 22,702 & 19,303 & 18,069 & 20,272 & 28,362 & 22,809 & 19,303 & 9,125 & 20,985 \\
\hline 2 YR OLD & 30,738 & 25,796 & 19,134 & 25,549 & 25,796 & 22,697 & 23,404 & 27,718 & 25,823 & 25,796 & & 24,177 \\
\hline 3 YR OLD & 18,087 & 16,194 & 24,347 & 20,215 & 17,886 & 20,992 & 25,553 & 17,924 & 20,491 & 16,194 & & 20,981 \\
\hline 4 YR OLD & 11,506 & 11,854 & 24,957 & 17,641 & 14,889 & 17,554 & 19,544 & 17,490 & 14,565 & 11,438 & & 18,282 \\
\hline 5 YR OLD & 21,705 & 14,490 & 15,853 & 12,414 & 12,414 & 16,932 & 12,312 & 20,946 & 11,373 & 12,414 & & 15,416 \\
\hline 6 YR OLD & 11,771 & 11,677 & 22,714 & 69,532 & 11,677 & 17,141 & 13,702 & 14,425 & 14,648 & 24,972 & 40,000 & 18,315 \\
\hline 7 YR OLD & 18,476 & 18,476 & 15,668 & 18,476 & 18,476 & 17,265 & 14,863 & 21,586 & 19,825 & 18,476 & & 18,484 \\
\hline 8 YR OLD & 13,598 & 10,395 & 10,377 & 10,395 & 10,990 & 12,097 & 15,901 & 44,127 & 10,463 & 12,334 & & 17,542 \\
\hline 9 YR OLD & 9,571 & 9,571 & 10,294 & 9,571 & 26,698 & 9,949 & 15,755 & 11,895 & 7,772 & 9,571 & & 12,757 \\
\hline 10 + YR OLD & 4,127 & 3,372 & 3,354 & 3,698 & 4,215 & 4,380 & 4,159 & 3,931 & 2,861 & 3,436 & 4,698 & 3,621 \\
\hline ALL VEHICLES & 7,441 & 5,587 & 7,787 & 8,519 & 5,961 & 5,897 & 7,429 & 8,589 & 3,665 & 5,003 & 8,943 & 5,993 \\
\hline
\end{tabular}

SIZE=HEAVY, ENGTYP=DIESEL

\begin{tabular}{|c|c|c|c|c|c|c|c|c|c|c|c|c|}
\hline \multicolumn{13}{|c|}{ SECTOR \# } \\
\hline AGE & 1 & 2 & 3 & 4 & 5 & 6 & 7 & 8 & 9 & 10 & 11 & $12^{*}$ \\
\hline NEW & 77,811 & 76,437 & 89,514 & 95,172 & 73,686 & 53,115 & 75,790 & 76,648 & 83,746 & 65,540 & 37,368 & 76,975 \\
\hline 1 YR OLD & 88,901 & 94,546 & 101,573 & 95,213 & 78,884 & 54,481 & 91,335 & 92,723 & 93,090 & 87,861 & 20,868 & 87,228 \\
\hline 2 YR OLD & 74,357 & 90,114 & 89,931 & 89,675 & 82,257 & 51,886 & 76,545 & 84,715 & 86,819 & 77,952 & 30,349 & 78,881 \\
\hline 3 YR OLD & 76,419 & 79,479 & 86,117 & 87,380 & 76,823 & 50,739 & 75,494 & 71,577 & 76,404 & 63,647 & 33,456 & 73,133 \\
\hline 4 YR OLD & 71,898 & 76,413 & 79,590 & 86,853 & 67,906 & 51,344 & 63,920 & 76,116 & 71,344 & 61,886 & 16,342 & 69,836 \\
\hline 5 YR OLD & 67,605 & 70,364 & 69,135 & 81,896 & 62,167 & 50,013 & 53,221 & 61,245 & 63,397 & 57,581 & 39,942 & 61,818 \\
\hline 6 YR OLD & 65,911 & 65,637 & 59,115 & 81,574 & 55,983 & 42,779 & 56,183 & 59,628 & 60,230 & 45,671 & 15,464 & 57,005 \\
\hline 7 YR OLD & 48,758 & 54,334 & 56,760 & 56,637 & 52,152 & 38,348 & 51,778 & 51,352 & 52,069 & 48,742 & 16,997 & 49,640 \\
\hline 8 YR OLD & 47,830 & 61,102 & 55,883 & 60,958 & 44,580 & 34,774 & 46,925 & 50,791 & 48,404 & 50,149 & 18,291 & 46,816 \\
\hline 9 YR OLD & 42,959 & 60,443 & 53,933 & 53,031 & 40,340 & 31,957 & 34,917 & 43,110 & 41,716 & 56,186 & 12,401 & 40,624 \\
\hline 10 + YR OLD & 23,297 & 37,276 & 31,463 & 36,816 & 22,669 & 20,398 & 19,726 & 31,250 & 20,658 & 28,819 & 12,788 & 22,890 \\
\hline ALL VEHICLES & 56,991 & 65,127 & 72,111 & 76,523 & 50,346 & 33,354 & 47,896 & 62,184 & 46,937 & 48,884 & 18,990 & 51,484 \\
\hline
\end{tabular}

* VMT for Government trucks is the average of all sectors within that size class and fuel type. 
SIZE=HEAVY, ENGTYP=LPG

\begin{tabular}{|c|c|c|c|c|c|c|c|c|c|c|c|c|}
\hline & $\overline{\text { CTOR \# }}$ & & & & & & & & & & & \\
\hline AGE & 1 & 2 & 3 & 4 & 5 & 6 & 7 & 8 & 9 & 10 & 11 & $12^{*}$ \\
\hline NEW & & & & & & & & & & & & 4,066 \\
\hline 1 YR OLD & 68,740 & & 91,744 & & 20,001 & 21,663 & 10,112 & & & & & 58,822 \\
\hline 2 YR OLD & & & & & & & 49,906 & & 25,807 & & & 39,705 \\
\hline 3 YR OLD & & & & & & 11,504 & & & & & & 12,046 \\
\hline 4 YR OLD & & & & & & & & & & & & 29,535 \\
\hline 5 YR OLD & 12,789 & 12,789 & 12,789 & 12,789 & 12,789 & 12,789 & 12,789 & 12,789 & 7,709 & 12,789 & & 10,501 \\
\hline 6 YR OLD & & & & & & & & & & & & 5,495 \\
\hline 7 YR OLD & & & & & & & & & & & & 54,980 \\
\hline 8 YR OLD & 15,055 & 15,055 & 15,055 & 15,055 & 15,055 & 15,055 & 15,055 & 15,055 & 14,921 & 18,632 & & 15,067 \\
\hline 9 YR OLD & & & & & & & & 21,296 & & & & 18,034 \\
\hline 10 + YR OLD & 3,713 & 4,318 & 4,313 & 4,315 & 7,338 & 2,043 & 4,322 & 4,311 & 6,615 & 4,304 & 2,960 & 4,635 \\
\hline ALL VEHICLES & 23,185 & 4,451 & 51,729 & 9,608 & 11,387 & 6,943 & 34,643 & 4,453 & 6,660 & 4,516 & 2,960 & 16,929 \\
\hline \multicolumn{13}{|c|}{ SIZE=HEAVY, ENGTYP=CNG } \\
\hline & CTOR \# & & & & & & & & & & & \\
\hline AGE & 1 & 2 & 3 & 4 & 5 & 6 & 7 & 8 & 9 & 10 & 11 & $12^{\star}$ \\
\hline NEW & & & & & & & & & & & & 42,807 \\
\hline 1 YR OLD & 605 & 605 & 605 & 605 & 605 & 683 & 608 & 605 & 33,048 & 605 & & 16,803 \\
\hline 2 YR OLD & & & 6,253 & & & & 7,471 & & 25,807 & & & 6,352 \\
\hline 3 YR OLD & & & & & & 11,504 & & & & & & 26,574 \\
\hline 4 YR OLD & & & & & & & & & & & 13,000 & 13,021 \\
\hline 5 YR OLD & 12,789 & 12,789 & 12,789 & 12,789 & 12,789 & 12,789 & 12,789 & 12,789 & 7,709 & 12,789 & & 10,501 \\
\hline 6 YR OLD & & & & & & & & & & & & 7,500 \\
\hline 7 YR OLD & & & & & & & & & & & & 16,351 \\
\hline 8 YR OLD & 4,793 & 4,793 & 4,793 & 4,793 & 18,216 & 4,793 & 4,793 & 4,793 & 8,695 & 24,674 & & 17,912 \\
\hline 9 YR OLD & & & & & & & & 21,296 & & & & 13,701 \\
\hline 10 + YR OLD & 2,055 & 228 & 227 & 224 & 297 & 2,397 & 2,959 & 224 & 3,724 & 250 & & 3,072 \\
\hline ALL VEHICLES & 9,118 & 380 & 4,170 & 1,060 & 9,071 & 2,327 & 2,845 & 10,714 & 6,837 & 496 & 16,497 & 7,366 \\
\hline
\end{tabular}

${ }^{*}$ VMT for Government trucks is the average of all sectors within that size class and fuel type. 
SIZE=MEDIUM, ENGTYP=GASOLINE

\begin{tabular}{|c|c|c|c|c|c|c|c|c|c|c|c|c|}
\hline \multicolumn{13}{|c|}{ SECTOR \# } \\
\hline AGE & 1 & 2 & 3 & 4 & 5 & 6 & 7 & 8 & 9 & 10 & 11 & $12^{*}$ \\
\hline NEW & 20,130 & 15,670 & 11,701 & 14,818 & 9,974 & 15,424 & 19,808 & 16,118 & 14,088 & 15,670 & 26,822 & 16,911 \\
\hline 1 YR OLD & 15,952 & 26,531 & 19,009 & 25,545 & 16,222 & 15,864 & 16,276 & 17,078 & 17,020 & 16,222 & 15,150 & 16,962 \\
\hline 2 YR OLD & 29,629 & 19,623 & 20,408 & 19,967 & 17,085 & 16,619 & 19,673 & 19,506 & 14,476 & 14,030 & 15,244 & 18,914 \\
\hline 3 YR OLD & 29,160 & 51,447 & 18,659 & 21,884 & 21,177 & 17,585 & 16,407 & 15,633 & 20,321 & 23,699 & 10,758 & 17,543 \\
\hline 4 YR OLD & 20,752 & 18,786 & 16,599 & 21,523 & 9,630 & 20,083 & 19,570 & 22,021 & 17,573 & 18,565 & 9,796 & 18,994 \\
\hline 5 YR OLD & 17,905 & 21,001 & 26,892 & 29,379 & 15,100 & 15,016 & 20,084 & 24,622 & 22,485 & 14,631 & 12,253 & 20,322 \\
\hline 6 YR OLD & 16,354 & 12,098 & 18,715 & 21,387 & 12,396 & 15,501 & 14,228 & 15,647 & 14,982 & 16,398 & 10,480 & 14,662 \\
\hline 7 YR OLD & 11,091 & 10,028 & 15,404 & 9,395 & 10,996 & 10,548 & 18,644 & 18,725 & 12,301 & 22,408 & 14,512 & 15,649 \\
\hline 8 YR OLD & 10,724 & 12,033 & 12,432 & 9,821 & 11,007 & 9,402 & 12,608 & 17,816 & 10,822 & 7,709 & 8,067 & 12,302 \\
\hline 9 YR OLD & 10,380 & 17,647 & 14,355 & 14,599 & 14,247 & 10,283 & 11,735 & 14,427 & 10,842 & 13,571 & 14,374 & 12,580 \\
\hline $10+$ YR OLD & 5,157 & 7,063 & 8,150 & 7,545 & 6,991 & 5,764 & 7,134 & 7,941 & 3,487 & 4,645 & 7,223 & 5,515 \\
\hline ALL VEHICLES & 8,802 & 10,959 & 11,606 & 12,203 & 8,729 & 7,787 & 10,698 & 12,938 & 4,650 & 6,609 & 10,784 & 8,563 \\
\hline \multicolumn{13}{|c|}{ SIZE=MEDIUM, ENGTYP=DIESEL } \\
\hline & CTOR \# & & & & & & & & & & & \\
\hline AGE & 1 & 2 & 3 & 4 & 5 & 6 & 7 & 8 & 9 & 10 & 11 & $12^{*}$ \\
\hline NEW & 33,001 & 34,807 & 29,314 & 30,165 & 23,460 & 22,816 & 25,509 & 31,621 & 23,361 & 22,283 & 12,971 & 26,681 \\
\hline 1 YR OLD & 37,815 & 41,761 & 29,606 & 40,925 & 24,818 & 23,516 & 30,217 & 33,621 & 26,584 & 27,038 & 13,967 & 29,605 \\
\hline 2 YR OLD & 33,561 & 31,723 & 29,908 & 30,954 & 27,254 & 25,937 & 28,633 & 37,170 & 31,824 & 36,253 & 16,515 & 29,858 \\
\hline 3 YR OLD & 43,518 & 38,556 & 29,283 & 38,743 & 25,189 & 25,918 & 28,114 & 36,215 & 31,381 & 20,526 & 11,169 & 30,333 \\
\hline 4 YR OLD & 31,118 & 26,651 & 27,510 & 38,386 & 23,808 & 19,427 & 24,333 & 29,901 & 28,926 & 32,979 & 14,031 & 25,890 \\
\hline 5 YR OLD & 37,790 & 41,633 & 30,520 & 41,112 & 22,039 & 24,801 & 23,540 & 32,409 & 23,082 & 77,549 & 14,746 & 27,675 \\
\hline 6 YR OLD & 17,462 & 19,142 & 25,328 & 46,341 & 24,076 & 22,313 & 17,146 & 24,976 & 22,830 & 18,066 & 11,737 & 21,029 \\
\hline 7 YR OLD & 24,401 & 19,893 & 24,247 & 17,841 & 25,775 & 15,595 & 21,560 & 18,258 & 23,131 & 35,106 & 12,906 & 19,979 \\
\hline 8 YR OLD & 13,724 & 22,494 & 20,136 & 36,958 & 18,170 & 11,942 & 15,869 & 16,164 & 18,099 & 16,903 & 12,649 & 16,452 \\
\hline 9 YR OLD & 22,621 & 15,060 & 19,747 & 50,788 & 13,262 & 14,753 & 17,166 & 18,705 & 14,783 & 16,944 & 11,313 & 17,677 \\
\hline 10 + YR OLD & 11,530 & 18,618 & 15,738 & 17,780 & 14,800 & 9,113 & 12,197 & 13,228 & 9,029 & 8,425 & 8,339 & 11,642 \\
\hline ALL VEHICLES & 24,509 & 26,321 & 23,849 & 32,153 & 19,903 & 15,749 & 21,013 & 25,159 & 17,405 & 20,318 & 12,252 & 20,833 \\
\hline
\end{tabular}

*VMT for Government trucks is the average of all sectors within that size class and fuel type. 
SIZE $=$ MEDIUM, ENGTYP=LPG

\begin{tabular}{|c|c|c|c|c|c|c|c|c|c|c|c|c|}
\hline \multicolumn{13}{|c|}{ SECTOR \# } \\
\hline AGE & 1 & 2 & 3 & 4 & 5 & 6 & 7 & 8 & 9 & 10 & 11 & $12^{*}$ \\
\hline NEW & 26,576 & & 19,270 & & 14,002 & 28,481 & 21,523 & & & & & 15,621 \\
\hline 1 YR OLD & 31,374 & & 24,895 & & 29,291 & & & & & & & 27,203 \\
\hline 2 YR OLD & 21,368 & 25,737 & 27,940 & 20,208 & 24,048 & 17,974 & 14,682 & 45,140 & 19,825 & 25,737 & & 24,048 \\
\hline 3 YR OLD & 10,376 & 33,137 & 36,704 & 33,137 & 18,079 & 29,973 & 20,777 & 34,316 & 33,137 & 33,137 & 16,562 & 19,644 \\
\hline 4 YR OLD & 62,039 & 62,039 & 30,814 & 62,039 & 23,259 & 25,729 & 45,587 & 61,903 & 62,039 & 62,039 & 18,649 & 26,566 \\
\hline 5 YR OLD & 25,923 & 109,778 & 15,996 & 109,778 & 18,806 & 42,881 & 58,088 & 75,157 & 109,778 & 109,778 & & 26,172 \\
\hline 6 YR OLD & 10,296 & 10,296 & 21,042 & 10,296 & 17,839 & 9,322 & 10,350 & 10,387 & 10,324 & 10,296 & & 17,257 \\
\hline 7 YR OLD & & & & & & & 15,780 & & & & & 13,217 \\
\hline 8 YR OLD & 15,085 & 1,538 & 12,227 & 1,538 & 26,841 & 8,727 & 44,891 & 6,023 & 3,004 & 1,538 & & 22,770 \\
\hline 9 YR OLD & 19,660 & 7,483 & 14,020 & 7,483 & 12,187 & 24,914 & 9,626 & 10,241 & 3,293 & 7,483 & & 14,205 \\
\hline $10+$ YR OLD & 6,948 & 9,059 & 4,922 & 8,946 & 7,979 & 5,886 & 4,647 & 8,884 & 5,152 & 2,693 & 25,209 & 7,114 \\
\hline ALL VEHICLES & 12,309 & 9,440 & 19,950 & 8,324 & 16,828 & 7,626 & 7,824 & 9,971 & 5,400 & 3,513 & 23,804 & 13,818 \\
\hline \multicolumn{13}{|c|}{ SIZE=MEDIUM, ENGTYP=CNG } \\
\hline & $\overline{\mathrm{CTOR} \#}$ & & & & & & & & & & & \\
\hline AGE & 1 & 2 & 3 & 4 & 5 & 6 & 7 & 8 & 9 & 10 & 11 & $12^{*}$ \\
\hline NEW & 26,576 & & 37,771 & 8,743 & 31,155 & 28,481 & 21,523 & & & & & 8,350 \\
\hline $\begin{array}{l}1 \text { YR OLD } \\
2 \text { YR OLD }\end{array}$ & 25,737 & 25,737 & 26,441 & 20,208 & 25,737 & 17,974 & 14,682 & 45,140 & 19,825 & 25,737 & & $\begin{array}{l}12,050 \\
26,072\end{array}$ \\
\hline 3 YR OLD & 64,291 & 60,658 & 60,658 & 60,658 & 41,370 & 40,546 & 50,347 & 62,387 & 60,658 & 60,658 & & 53,314 \\
\hline 4 YR OLD & 62,039 & 62,039 & 69,629 & 62,039 & 16,438 & 25,729 & 45,587 & 61,903 & 62,039 & 62,039 & 5,973 & 35,678 \\
\hline 5 YR OLD & 50,094 & 109,778 & 109,778 & 109,778 & 109,778 & 42,881 & 58,088 & 75,157 & 109,778 & 109,778 & & 1,157 \\
\hline 6 YR OLD & & & & & 37,549 & & 37,353 & 30,632 & 27,751 & & & 7,084 \\
\hline 7 YR OLD & & & & & & & 33,190 & & & & & 19,216 \\
\hline 8 YR OLD & 19,778 & 19,778 & 19,778 & 19,778 & 5,369 & 19,764 & 19,778 & 19,345 & 19,510 & 19,778 & & 8,017 \\
\hline 9 YR OLD & 5,706 & 7,483 & 14,020 & 7,483 & 7,483 & 24,914 & 9,626 & 10,241 & 6,245 & 7,483 & & 12,454 \\
\hline 10 + YR OLD & 8,067 & 2,048 & 2,106 & 1,994 & 2,778 & 9,686 & 4,323 & 8,545 & 4,098 & 3,448 & 9,046 & 5,341 \\
\hline ALL VEHICLES & 9,227 & 3,597 & 11,858 & 8,151 & 5,150 & 10,472 & 5,262 & 9,209 & 4,504 & 3,502 & 3,300 & 6,231 \\
\hline
\end{tabular}

*VMT for Government trucks is the average of all sectors within that size class and fuel type. 
VIUS 1997

TOTAL VMT FOR 1997 BY SECTOR

\begin{tabular}{|c|c|c|c|}
\hline & SECTOR & & VMT \\
\hline SECTOR & \# & TOTAL VMT & SHARES \\
\hline CHEMICALS,RUBBER,PLASTIC & 01 & $8,878,079,000$ & $5.9 \%$ \\
\hline PRIMARY METALS & 02 & $5,285,467,986$ & $3.5 \%$ \\
\hline PROCESSED FOODS & 03 & $26,031,042,817$ & $17.2 \%$ \\
\hline PAPER PRODUCTS & 04 & $7,043,457,781$ & $4.6 \%$ \\
\hline PETROLEUM PRODUCTS & 05 & $5,925,258,193$ & $3.9 \%$ \\
\hline STONE,CLAY,GLASS,CONCRETE & 06 & $20,488,067,721$ & $13.5 \%$ \\
\hline METAL DURABLE & 07 & $24,373,313,080$ & $16.1 \%$ \\
\hline OTHER MANUFACTURING & 08 & $25,200,330,240$ & $16.6 \%$ \\
\hline AGRICULTURE & 09 & $24,451,510,369$ & $16.1 \%$ \\
\hline MINING & 10 & $1,793,901,816$ & $1.2 \%$ \\
\hline UTILITY & 11 & $2,174,751,254$ & $1.4 \%$ \\
\hline GOVERNMENT** & 12 & $6,825,268,839$ & $4.5 \%$ \\
\hline TOTAL & & $151,645,180,257$ & $100.0 \%$ \\
\hline
\end{tabular}

* Government VMT was estimated by multiplying the number of medium government trucks by the average annual VIUS VMT for medium trucks, plus the number of heavy government trucks by the average annual VIUS VMT for heavy trucks. 


\begin{tabular}{lcrrr}
\hline & SECTOR & MEDIUM TRUCKS & HEAVY TRUCKS & \multicolumn{2}{c}{ MEDIUM \&HEAVY } \\
SECTOR & \multicolumn{1}{c}{ M } & \multicolumn{1}{c}{ TOTAL VMT } & \multicolumn{1}{c}{ TOTAL VMT } & \multicolumn{1}{c}{ TOTAL VMT } \\
\hline CHEMICALS,RUBBER,PLASTIC & 01 & $2,094,397,195$ & $6,783,681,805$ & $8,878,079,000$ \\
PRIMARY METALS & 02 & $816,041,837$ & $4,469,426,149$ & $5,285,467,986$ \\
PROCESSED FOODS & 03 & $5,058,570,001$ & $20,972,472,816$ & $26,031,042,817$ \\
PAPER PRODUCTS & 04 & $1,146,082,330$ & $5,897,375,451$ & $7,043,457,781$ \\
PETROLEUM PRODUCTS & 05 & $1,662,847,830$ & $4,262,410,364$ & $5,925,258,193$ \\
STONE,CLAY,GLASS,CONCRETE & 06 & $4,381,794,005$ & $16,106,273,716$ & $20,488,067,721$ \\
METAL DURABLE & 07 & $8,393,125,646$ & $15,980,187,434$ & $24,373,313,080$ \\
OTHER MANUFACTURING & 08 & $7,434,150,669$ & $17,766,179,570$ & $25,200,330,240$ \\
AGRICULTURE & 09 & $5,043,708,879$ & $19,407,801,489$ & $24,451,510,369$ \\
MINING & 10 & $127,892,909$ & $1,666,008,907$ & $1,793,901,816$ \\
UTILITY & 11 & $1,779,640,028$ & $395,111,226$ & $2,174,751,254$ \\
GOVERNMENT* & 12 & $3,577,625,344$ & $3,247,643,495$ & $6,825,268,839$ \\
\hline TOTAL & & $158,470,449,095$ & $158,470,449,095$ & $151,645,180,257$ \\
\hline
\end{tabular}

* Government VMT was estimated by multiplying the number of medium government trucks by the average annual VIUS VMT for medium trucks, plus the number of heavy government trucks by the average annual VIUS VMT for heavy trucks. 
VIUS 1997

NEW TRUCKS BY FLEET INDICATOR, SECTOR, AND SIZE

FLEET INDICATOR=FLEET

\begin{tabular}{|c|c|c|c|c|c|c|c|}
\hline & \multicolumn{4}{|c|}{ rLELI IIVUIUATUR=ГL } & \multicolumn{3}{|c|}{ 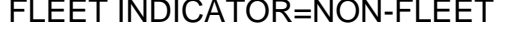 } \\
\hline SECTOR & SECTOR \# & HEAVY & MEDIUM & TOTAL & HEAVY & MEDIUM & $\overline{\text { TOTAL }}$ \\
\hline CHEMICALS,RUBBER,PLASTIC & 01 & 9,395 & 3,251 & 12,646 & 1,512 & 2,858 & 4,369 \\
\hline PRIMARY METALS & 02 & 4,821 & 566 & 5,388 & 1,112 & 541 & 1,653 \\
\hline PROCESSED FOODS & 03 & 33,959 & 11,286 & 45,245 & 5,322 & 3,431 & 8,753 \\
\hline PAPER PRODUCTS & 04 & 7,661 & 5,440 & 13,101 & 1,494 & 1,471 & 2,965 \\
\hline PETROLEUM PRODUCTS & 05 & 4,729 & 2,252 & 6,981 & 1,593 & 1,076 & 2,670 \\
\hline STONE,CLAY,GLASS,CONCRETE & 06 & 21,543 & 7,102 & 28,645 & 8,429 & 7,886 & 16,314 \\
\hline METAL DURABLE & 07 & 18,102 & 10,399 & 28,501 & 5,870 & 14,444 & 20,313 \\
\hline OTHER MANUFACTURING & 08 & 27,693 & 14,312 & 42,006 & 4,967 & 9,627 & 14,594 \\
\hline AGRICULTURE & 09 & 17,500 & 5,236 & 22,736 & 8,521 & 4,505 & 13,026 \\
\hline MINING & 10 & 2,453 & 140 & 2,593 & 913 & 254 & 1,167 \\
\hline UTILITY & 11 & 606 & 6,624 & 7,230 & 172 & 1,445 & 1,617 \\
\hline GOVERNMENT & 12 & 5,380 & 10,750 & 16,130 & & & \\
\hline TOTAL & & 153,842 & 77,359 & 231,201 & 39,905 & 47,538 & 87,443 \\
\hline
\end{tabular}


SHARE OF MILES IN URBAN AREAS, 1997 AND 1998

\begin{tabular}{|c|c|c|c|}
\hline & \multicolumn{2}{|c|}{ Urban miles Total miles } & Share \\
\hline & 1997 & 1997 & 1997 \\
\hline Medium Trucks & 31,836 & 66,893 & $47.59 \%$ \\
\hline Heavy Trucks & 44,943 & 124,584 & $36.07 \%$ \\
\hline & 1998 & 1998 & 1998 \\
\hline Medium Trucks & 33,122 & 67,894 & $48.78 \%$ \\
\hline Heavy Trucks & 47,856 & 128,159 & $37.34 \%$ \\
\hline
\end{tabular}

Source:

U.S. Department of Transportation, Federal Highway Administration, Highway Statistics 1998, Washington, DC, 1999, Table VM1, p. V47. 
VIUS 1997

FUEL ECONOMY (MPG) BY SIZE, FUEL TYPE AND AGE

SIZE=MEDIUM

SIZE=HEAVY

\begin{tabular}{lrrrrrrrr}
\hline AGE & GASOLINE & DIESEL & \multicolumn{1}{c}{ LPG } & \multicolumn{1}{c}{ CNG } & GASOLINE & DIESEL & \multicolumn{1}{c}{ LPG } & \multicolumn{1}{c}{ CNG } \\
\hline NEW & 8.55938 & 8.48462 & 5.14602 & 5.89747 & 9.09430 & 6.26224 & 7.54532 & 5.56074 \\
1 YR OLD & 9.15906 & 8.83929 & 5.69105 & & 8.59393 & 6.10237 & 4.75677 & 7.73772 \\
2 YR OLD & 8.48974 & 8.43387 & 5.82966 & 9.84996 & 8.08873 & 6.15891 & 7.06584 & 7.49896 \\
3 YR OLD & 8.80769 & 8.65505 & 6.35182 & 9.76057 & 8.51783 & 6.12948 & 7.99987 & 5.50000 \\
4 YR OLD & 8.97485 & 8.38702 & 5.96608 & 7.97772 & 6.85549 & 6.11884 & 8.00000 & 10.49809 \\
5 YR OLD & 8.72005 & 8.25991 & 4.82901 & 5.01227 & 7.89165 & 6.29103 & 7.70000 & 7.70000 \\
6 YR OLD & 8.63522 & 8.22619 & 6.21448 & 7.60000 & 5.92985 & 5.98831 & 5.00000 & \\
7 YR OLD & 8.42333 & 8.36816 & 6.03151 & 4.79054 & 8.02504 & 5.99802 & 4.00028 & 6.48052 \\
8 YR OLD & 8.39867 & 8.91005 & 7.43286 & 6.81131 & 8.34495 & 5.86418 & 3.50468 & 5.90000 \\
9 YR OLD & 8.39935 & 8.12454 & 5.10676 & 7.75425 & 7.63721 & 5.74797 & 6.53280 & 4.00387 \\
10 + YR OLD & 7.76439 & 7.67455 & 6.65446 & 7.11279 & 7.09995 & 5.60765 & 5.88435 & 5.34362 \\
\hline TOTAL & 7.99135 & 8.23274 & 6.32660 & 6.67219 & 7.23639 & 5.91670 & 5.87161 & 6.10575 \\
\hline
\end{tabular}

${ }^{* *}$ Following the previous methodology, medium trucks with fuel economy greater than 13 and heavy trucks with fuel economy greater than 11 were not used in these calculations. 


\section{INTERNAL DISTRIBUTION}

1. G. E. Courville

2. T. R. Curlee

3. S. C. Davis

4. P. S. Hu

5. C. I. Moser
6. R. B. Shelton

7. Central Research Library

8. Document Reference Section

9. Laboratory Records

\section{EXTERNAL DISTRIBUTION}

10. L. A. Abron, President, PEER Consultants, P.C., 1460 Gulf Blvd., $11^{\text {th }}$ Floor, Clearwater, Florida 34630

11. S. L. Cutter, Director, Hazards Research Lab, Department of Geography, University of South Carolina, Columbia, South Carolina 29208

12. S. G. Hildebrand, Director, Environmental Sciences Division, Oak Ridge National Laboratory, Post Office Box 2008, Oak Ridge, Tennessee 37831-6037

13. P. R. Rittelmann, FAIA, Executive Vice President, Burt Hill Kosar Rittelmann Associates, 400 Morgan Center, Butler, Pennsylvania 16001-5977

14. S. F. Tierney, The Economic Resource Group, Inc., One Mifflin Place, Cambridge, Massachusetts 02138

15. C. M. Walton, Ernest H. Cockrell Centennial Chair in Engineering and Chairman, Department of Civil Engineering, University of Texas at Austin,E Cockrell, Jr. Hall I, Suite 4210, Austin, Texas 78712-1075

16-20. Center for Transportation Analysis, Energy Division, Building 3156, Room 211. 